\title{
Evaluation and Enhancement of Carbon Dioxide Flooding Through Sweep Improvement
}

\section{Final Technical Report}

Project Period

October 1, 2004 through September 30, 2009

\section{Principal Author}

Richard G. Hughes, Louisiana State University

April 2010

DE-FC26-04NT15536

Louisiana State University

Office of Sponsored Programs

330 Thomas Boyd Hall

Baton Rouge, LA 70803 


\section{DISCLAIMER}

This report was prepared as an account of work sponsored by an agency of the United States Government. Neither the United States Government nor any agency thereof, nor any of their employees, makes any warranty, express or implied, or assumes any legal liability or responsibility for the accuracy, completeness, or usefulness of any information, apparatus, product, or process disclosed, or represents that its use would not infringe privately owned rights. Reference herein to any specific commercial product, process, or service by trade name, trademark, manufacturer, or otherwise does not necessarily constitute or imply its endorsement, recommendation, or favoring by the United States Government or any agency thereof. The views and opinions of authors expressed herein do not necessarily state or reflect those of the United States Government or any agency thereof. 


\section{Table of Contents}

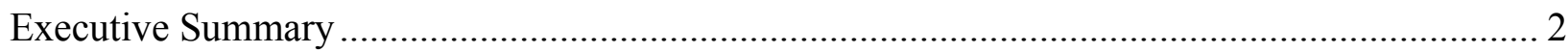

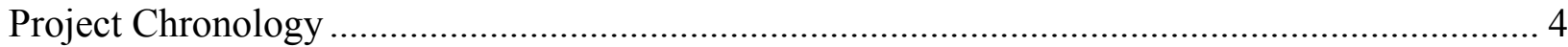

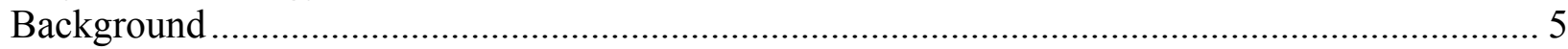

Task 1: Historical Evaluation of Sweep Efficiency in a Mature $\mathrm{CO}_{2}$ Flood ................................. 8

Subtask 1.1 Evaluation of Light Oil Displacement by $\mathrm{CO}_{2}$ Flooding ....................................... 8

Subtask 1.2: Study of $\mathrm{CO}_{2}$ Conformance and Sweep Efficiency .......................................... 12

Evaluation of Interwell Connectivity in $\mathrm{CO}_{2}$ Flooding.................................................... 12

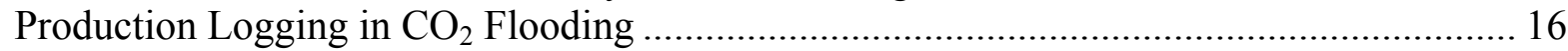

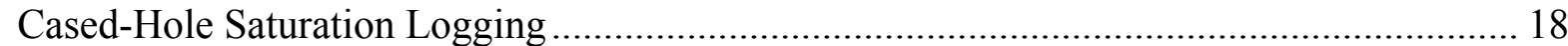

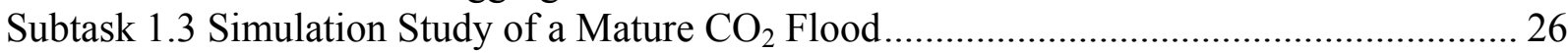

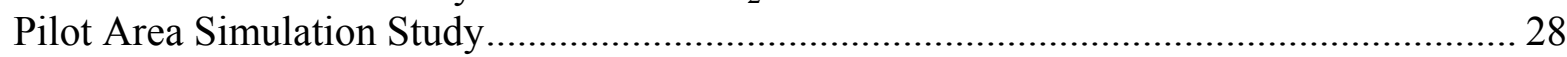

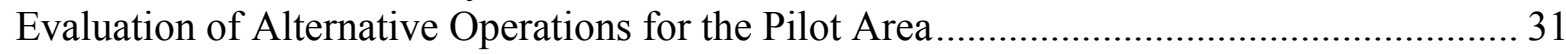

Currently Active Area Simulation Study ....................................................................... 36

Task 2: Extension of Sweep Efficiency Findings to Target Reservoirs for $\mathrm{CO}_{2}$ Flooding .......... 42

Subtask 2.1 Light Oil Displacement by $\mathrm{CO}_{2}$ Flooding …………………………….......... 42

Subtask 2.2 Evaluation of Heavy Oil Displacement by $\mathrm{CO}_{2}$ Flooding ..................................... 46

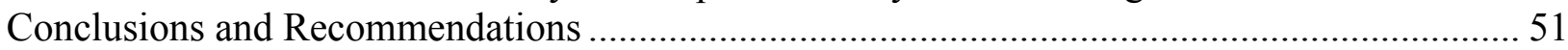

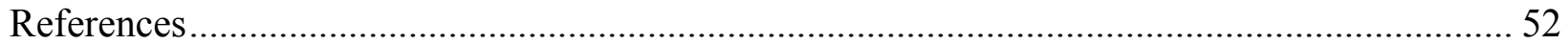




\section{Executive Summary}

\section{Evaluation and Enhancement of Carbon Dioxide Flooding Through Sweep Improvement DE-FC26-04NT15536}

Carbon dioxide displacement is a common improved recovery method applied to light oil reservoirs $\left(30-45^{\circ} \mathrm{API}\right)$. The economic and technical success of $\mathrm{CO}_{2}$ floods is often limited by poor sweep efficiency or large $\mathrm{CO}_{2}$ utilization rates. Projected incremental recoveries for $\mathrm{CO}_{2}$ floods range from $7 \%$ to $20 \%$ of the original oil in place; however, actual incremental recoveries range from $9 \%$ to $15 \%$ of the original oil in place, indicating the potential for significant additional recoveries with improved sweep efficiency.

This research program was designed to study the effectiveness of carbon dioxide flooding in a mature reservoir to identify and develop methods and strategies to improve oil recovery in carbon dioxide floods. Specifically, the project has focused on relating laboratory, theoretical and simulation studies to actual field performance in a $\mathrm{CO}_{2}$ flood in an attempt to understand and mitigate problems of areal and vertical sweep efficiency. In this work the focus has been on evaluating the status of existing swept regions of a mature $\mathrm{CO}_{2}$ flood and developing procedures to improve the design of proposed floods. The Little Creek Field, Mississippi has been studied through laboratory, theoretical, numerical and simulation studies in an attempt to relate performance predictions to historical reservoir performance to determine sweep efficiency, improve the understanding of the reservoir response to $\mathrm{CO}_{2}$ injection, and develop scaling methodologies to relate laboratory data and simulation results to predicted reservoir behavior.

Existing laboratory information from Little Creek was analyzed and an extensive amount of field data was collected. This was merged with an understanding of previous work at Little Creek to generate a detailed simulation study of two portions of the field - the original pilot area and a currently active part of the field. This work was done to try to relate all of this information to an understanding of where the $\mathrm{CO}_{2}$ went or is going and how recovery might be improved. New data was also generated in this process. Production logs were run to understand where the $\mathrm{CO}_{2}$ was entering the reservoir related to core and log information and also to corroborate the simulation model. A methodology was developed and successfully tested for evaluating saturations in a cased-hole environment. Finally an experimental and theoretical program was initiated to relate laboratory work to field scale design and analysis of operations.

This work found that an understanding of vertical and areal heterogeneity is crucial for understanding sweep processes as well as understanding appropriate mitigation techniques to improve the sweep. Production and injection logs can provide some understanding of that heterogeneity when core data is not available. The cased-hole saturation logs developed in the project will also be an important part of the evaluation of vertical heterogeneity. Evaluation of injection well/production well connectivities through statistical or numerical techniques were found to be as successful in evaluating $\mathrm{CO}_{2}$ floods as they are for waterfloods. These are likely to be the lowest cost techniques to evaluate areal sweep. Full field simulation and 4D seismic techniques are other possibilities but were beyond the scope of the project. Detailed simulation studies of pattern areas proved insightful both for doing a "post-mortem" analysis of the pilot area as well as a late-term, active portion of the Little Creek Field. This work also evaluated options for improving sweep in the current flood as well as evaluating options that could have been successful at recovering more oil. That simulation study was successful due to the 
integration of a large amount of data supplied by the operator as well as collected through the course of the project. While most projects would not have the abundance of data that Little Creek had, integration of the available data continues to be critical for both the design and evaluation stages of $\mathrm{CO}_{2}$ floods. For cases where data availability is limited, running injection/production logs and/or running cased-hole saturation tools to provide an indication of vertical heterogeneity will be important. 


\section{Project Chronology}

Carbon dioxide displacement is a common improved recovery method applied to light oil reservoirs. The economic and technical success of $\mathrm{CO}_{2}$ floods is often limited by poor sweep efficiency or large $\mathrm{CO}_{2}$ utilization rates. Projected incremental recoveries for $\mathrm{CO}_{2}$ floods range from $7 \%$ to $23 \%$ of the original oil in place. Actual incremental recoveries range from $9 \%$ to $15 \%$ of the original oil in place, indicating the potential for significant additional recoveries with improved sweep efficiency.

Denbury Resources, Inc., headquartered in Plano, Texas, has significant oil and gas operations in Mississippi and is one of the largest oil and gas producers in that state. Their operations include several reservoirs undergoing $\mathrm{CO}_{2}$ flooding. In addition, the company is expanding their $\mathrm{CO}_{2}$ flooding experience to other reservoirs and other states throughout the southern Gulf States of Louisiana, Alabama and East Texas by implementing new $\mathrm{CO}_{2}$ floods, re-evaluating current floods, and studying potential applications for $\mathrm{CO}_{2}$ sequestration.

The Mewbourne School of Petroleum and Geological Engineering at the University of Oklahoma partnered with Denbury Resources in 2004 to evaluate $\mathrm{CO}_{2}$ sweep and displacement efficiency in a mature oil reservoir. The Little Creek Field, Mississippi was chosen for study through data evaluation, laboratory experiments and simulated performance predictions compared to the historical reservoir performance to determine sweep efficiency, improve the understanding of the reservoir response to $\mathrm{CO}_{2}$ injection, and develop scaling methodologies to relate laboratory data and simulation results to predicted reservoir behavior.

The Department of Energy recognized the benefit of such a study and awarded a grant to this partnership and work commenced on October 1, 2004. A high pressure-high temperature PVT cell and a slim tube setup were to be the focal points of proposed experimental work. Researchers began the work of developing suitable experiments, collecting data and beginning simulation studies in support of the project. Theoretical work also began to investigate behindpipe saturation monitoring and laboratory to field scaling issues.

In August 2005 one of the principal investigators on the project joined the faculty at Louisiana State University and a sub-contract from OU to LSU was issued for the logging portion of the project and to keep him in an advisory role for the experimental and numerical work. This sub-contract was awarded in November 2005 and one of the students working on the project transferred from OU to LSU to continue his work. In early 2006, negotiations began to transfer the entire project to LSU due to the fact that the remaining investigator at OU left to take a position in industry. In order to complete the transfer, spending on both the original contract and on the sub-contract was suspended and students at OU were no longer working on the project and students at LSU were working part-time on the project as they were being paid through the use of State of Louisiana funds available through the LSU Craft and Hawkins Department of Petroleum Engineering. Completion of the transfer occurred retroactive to October 1, 2006, but official notification occurred in February 2007.

Through the negotiations the laboratory work was changed to focus more heavily on displacement studies as equipment to do displacement work was thought to be more readily available at LSU than the PVT equipment. In fact, all of the equipment at LSU was tied to other supported projects and a displacement laboratory had to be developed using spare equipment from other LSU labs supplemented by the purchase of a high pressure coreholder and a high pressure syringe pump. This has delayed the experimental work considerably; however, students paid through the grant for part of their studies continue to work to finish their tasks. 
Due both to the transfer delays, the timing of the transfer and extension notifications, and to the delays in getting the work completed, the Department of Energy extended the completion date of the project to September 30, 2009. Spending on the project ceased on that date but students continue to finish their work and papers based on the tasks related to the project continue to be published. In addition, Denbury Resources, Inc. continues to be a strong partner in evaluating methods to improve their displacement and sweep efficiencies in the field.

\section{Background}

The Little Creek Field was discovered by Shell Oil Company in January 1958, and is located in Lincoln and Pike Counties in southwest Mississippi (Figure 1). The producing pay zone is the lower Tuscaloosa (Upper Cretaceous) Denkman sand. The current operator designates the producing zones as the Q and the $\mathrm{Q}_{2}$ sandstones (Werren, et al., 1990).

The Little Creek Field originally contained an estimated 101.9 million barrels of oil (Cronquist, 1968; Hansen, 1977b). The primary drive mechanisms were said to be fluid expansion and solution gas drive with limited aquifer influx based on the early production data (Hansen, 1977a; Werren, et al., 1990). The field began to produce oil from the Shell-Lemann No. 1 well with 588 BOPD and 260 MCFGPD from an open-hole interval from 10,770 to 10,790 ft (Werren, et al., 1990). The field was rapidly developed by drilling on 40 acre spacing in the northern part of the field and field production was around 9100 BOPD from 56 wells at the end of 1958 (Cronquist, 1968). The discovery of the southern part of the field was in November, 1958. Through 1961, 190 wells had been completed with 155 producers. Werren, et al. (1990) state that through 1990 the total number of wells in the field was 208 with 162 being producing wells. There are a total of 233 wells in the field today (Pennell, 2007). Figure 2 shows the production history of the field.

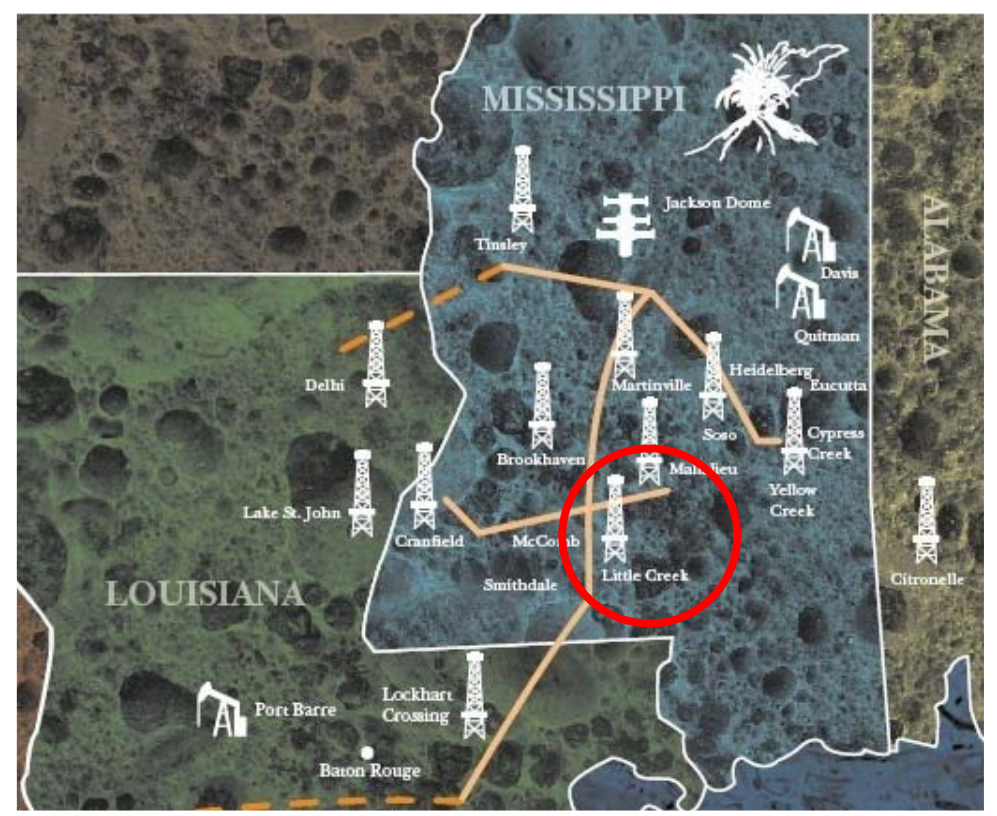

Figure 1: Little Creek Field Location (from Denbury Resources, Inc., 2007) 


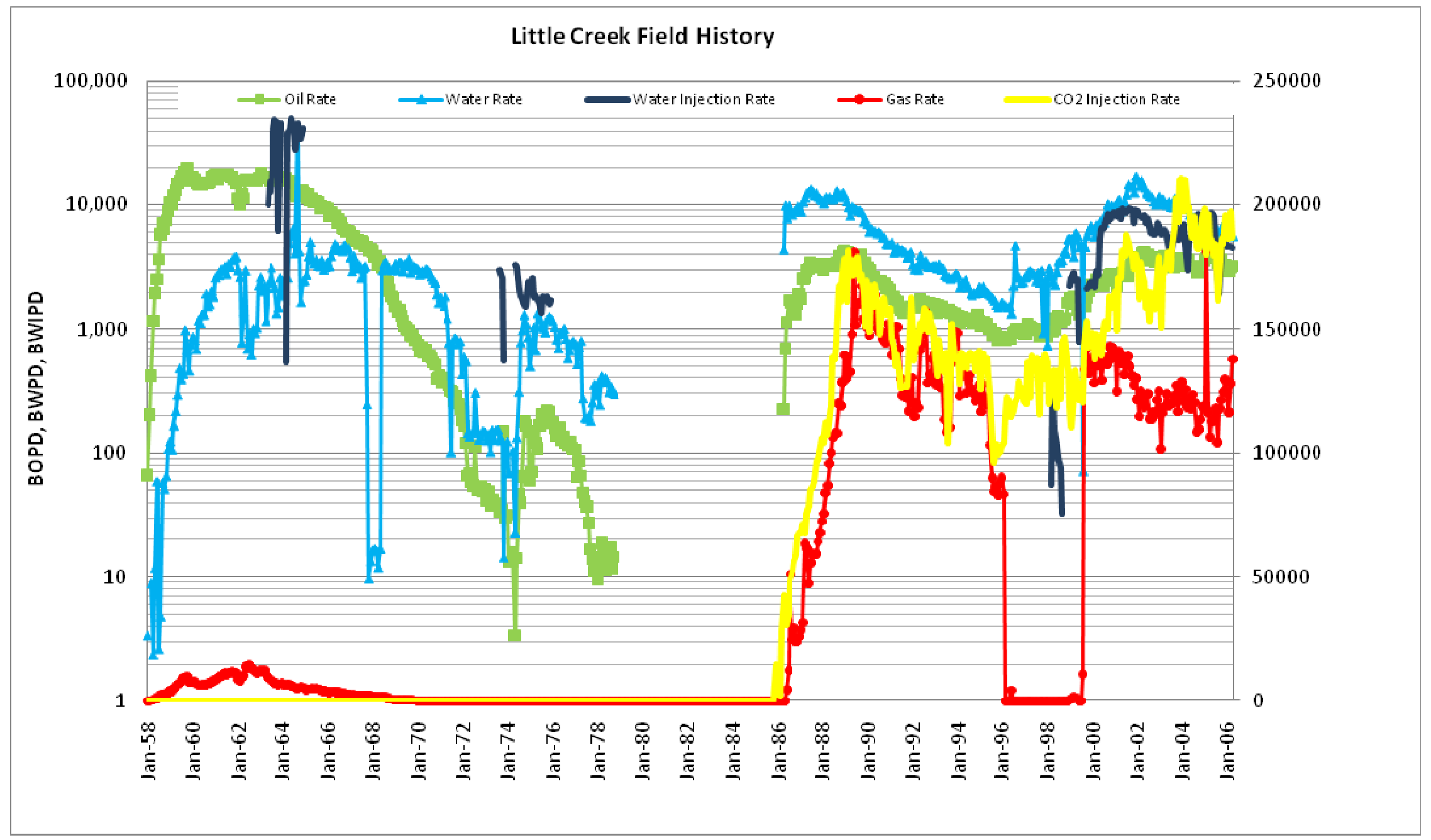

Figure 2: Field Historical Production and Injection Performance 
Primary recovery was approximately 25 million barrels of oil (MMBO) which was 25\% of the original oil in place (OOIP). A peripheral line-drive waterflood operation was initiated in early 1962 . Waterflooding was very successful with an additional $21.7 \mathrm{MMBO}(22 \%$ of the OOIP) produced during secondary recovery (Cronquist, 1968; Hansen, 1977a; Smith, 1973). Production decline began in 1964 and waterflooding was stopped in early 1970 (Cronquist, 1968). However, one well (Well 2-4A) produced oil until late 1978 even after waterflooding had ended.

Shell Oil Company considered different methodologies to recover the large amount of remaining oil considering that an estimated $47 \%$ of the OOIP was produced by primary and secondary means. They evaluated two miscible project options using reservoir simulation studies. A natural gas miscible displacement process was initially proposed, but they did not pursue this option due to the high amount of natural gas required (Hansen, 1977a). Shell decided to pursue $\mathrm{CO}_{2}$ flooding instead. $\mathrm{A} \mathrm{CO}_{2}$ pilot was performed between February 1974 and February 1977 and more than 120,000 bbls of oil (an additional $0.12 \%$ of the OOIP ) was produced (Hansen, 1977a). After a long shut-in period in the field due to the construction of the Jackson Dome $\mathrm{CO}_{2}$ pipeline and the field $\mathrm{CO}_{2}$ injection facilities, tertiary recovery was initiated in December, 1985 (Werren, et al., 1990). Since that time, $\mathrm{CO}_{2}$ has been continuously injected into the field, and an additional $18 \mathrm{MMBO}$ (18.4\% of OOIP) has been recovered.

Little Creek Field was operated by Shell Oil Company until J.P. Oil Company purchased the field in June, 1996. Denbury Resources, Inc. has been the operator of the field since the company acquired the field in September, 1999 (Senocak, et al., 2008). Subsequently, the company purchased the West Mallalieu, McComb and Brookhaven fields which have reservoir characteristics that are very similar to Little Creek and were short extensions to a $\mathrm{CO}_{2}$ pipeline running from the Jackson Dome area in south-central Mississippi. The existing $\mathrm{CO}_{2}$ flood at the West Mallalieu field was expanded and a flood was implemented in the McComb field. The Brookhaven field and a number of fields in eastern Mississippi, namely the Heidelberg, Eucutta, Quitman, Davis, Sandersville, Soso, Martinville and King Bee Fields are in various stages of development and response to $\mathrm{CO}_{2}$ injection.

All of these fields are highly channelized fluvial-deltaic sequences. Questions have been raised regarding the best flood pattern and operating strategy. Little Creek has sufficient data for evaluating this issue. The Little Creek Field provides an ideal "field laboratory" to study the historical performance of the reservoir and investigate the reservoir response to $\mathrm{CO}_{2}$ injection.

At Little Creek, inverted nine-spot pattern flooding is used for $\mathrm{CO}_{2}$ injection operations and production wells that have uneconomically high gas-oil ratios are converted to injection wells. The reservoir has been subjected to $\mathrm{CO}_{2}$ flooding for more than 20 years and was considered to be a good example for evaluating the flood performance of a late-in-life reservoir. Total recovery from the field is approximately $65 \%$ (calculated by $101.9 \mathrm{MMBO}$ of OOIP); thus the target for any further EOR operations is the remaining 35\%. The most important thing influencing the project economics for tertiary recovery processes is the amount of remaining oil. Denbury would certainly like to increase or accelerate recovery in Little Creek Field, but they also have other fields where they have $\mathrm{CO}_{2}$ operations. They are also extending their $\mathrm{CO}_{2}$ pipeline down into eastern Texas. This has the potential to significantly increase the recovery from fields in this area. Some of these fields will have characteristics similar to the Mississippi fields. Most of the East Texas fields are much more heterogeneous and much thicker reservoirs than their Mississippi counterparts which will make assessment and mitigation of sweep problems that much more important. Therefore, identifying strategies and modifications to 
current operations to improve recovery in a long-term flood such as Little Creek should be beneficial not only for Denbury but also to other operators considering $\mathrm{CO}_{2}$ floods.

As originally proposed, this project had two technical tasks specified with an additional reporting and technology transfer task. The technical tasks were: (1) Historical evaluation of sweep efficiency in a mature $\mathrm{CO}_{2}$ flood; (2) Extension of sweep efficiency findings to target reservoirs for $\mathrm{CO}_{2}$ flooding. These over-arching tasks were broken into subtasks. The subtasks for Task 1 were (a) an evaluation of light oil displacement by $\mathrm{CO}_{2}$ flooding, (b) a study of $\mathrm{CO}_{2}$ conformance and sweep efficiency and (c) a simulation study of a mature $\mathrm{CO}_{2}$ flood. All of these tasks were essentially a detailed study of the Little Creek Field. The subtasks for Task 2 were to extend the Task 1 findings to first light oil target reservoirs and second to heavy oil target reservoirs. Finally, the third task in the proposal was a reporting and technology transfer task which had stated deliverables of annual reports, technical papers and DOE briefings. Expansion of each of these tasks follows.

\section{Task 1: Historical Evaluation of Sweep Efficiency in a Mature $\mathrm{CO}_{2}$ Flood}

This task was devoted to analyzing the historical performance of the Little Creek Field operated by Denbury Resources, Inc. (DRI) to evaluate the effectiveness of injection and production operations during the active $\mathrm{CO}_{2}$ flooding process. This task was to focus on comparing performance predictions to actual historical performance data including displacement studies, conformance issues, and sweep efficiency. This "efficiency/effectiveness" comparison was to be used to propose methodologies and/or guidelines for implementing $\mathrm{CO}_{2}$ floods including ways to enhance sweep efficiency to increase oil recoveries. It was to do so through three subtasks. The three subtasks can be characterized by:

- A theoretical and experimental task to relate experimental results to field-scale processes (ongoing)

- Methods to evaluate conformance and sweep through the acquisition and analysis of well logs and through an evaluation of the available production and injection data (completed)

- A simulation study of the Little Creek Field to observe historical sweep effects and to use these observations to evaluate alternative options that might have improved sweep efficiency and hence recovery from the field (completed)

Each of these subtasks will be described in the following sections.

\section{Subtask 1.1 Evaluation of Light Oil Displacement by $\mathrm{CO}_{2}$ Flooding}

As proposed, laboratory studies were to be conducted using reservoir fluids and $\mathrm{CO}_{2}$ for assessment of reservoir displacement properties. Pressure-volume-temperature (PVT) analyses and slim tube experiments were to be conducted to develop techniques to relate laboratory scale estimates of recovery to field scale observations of sweep in Little Creek Field. With an improved understanding of how to relate laboratory studies to predictions of actual reservoir response it was hoped that operators would be more confident in undertaking $\mathrm{CO}_{2}$ floods.

Laboratory studies changed from the PVT and slim tube studies due to the equipment at OU, to displacement studies with the transfer to LSU. Displacement experiments were designed and built to test displacement efficiency of continuous $\mathrm{CO}_{2}$ injection, water-alternating-gas injection, simultaneous water and $\mathrm{CO}_{2}$ injection and alternating $\mathrm{CO}_{2}$-foam injection. 
The experiments are being conducted using 1 inch diameter by 12 inch cylindrical Berea sandstone cores (Cleveland Quarries, Ohio), n-decane colored with Sudan-4 dye as the oleic phase, and supercritical $\mathrm{CO}_{2}$ as the tertiary recovery fluid. The aqueous phase brine is a $2 \%$ (by weight) $\mathrm{NaCl}$ solution. The n-decane was chosen as the oleic phase as it is neutral towards inducing wettability changes in the rock. The minimum miscibility pressure for the fluid combination was estimated to be $1880 \mathrm{psi}$ at $160^{\circ} \mathrm{F}$ and about 1000 psi at $82^{\circ} \mathrm{F}$ (Kulkarni, 2003). All floods are conducted at atmospheric temperature and pressure values greater than 1800 psi to ensure miscibility between $\mathrm{CO}_{2}$ and n-decane.

A Teledyne ISCO Model-260 D syringe pump (Figure 3) was purchased for the project and is being used for the injection of various fluids. The flow range of the pump is 0.001 to $107.00 \mathrm{ml} / \mathrm{min}$ and the pressure range is between 0 and $7500 \mathrm{psi}$. A TEMCO, Inc pressure tapped Hassler type coreholder with a VITON sleeve (Figure 4) was also purchased for the project and is used to contain the experiment to the core and to minimize any damage to equipment caused by the $\mathrm{CO}_{2}$.

A schematic of the experimental setup is shown in Figure 5. The setup consists of the dual syringe pump coupled with a continuous flow valve package. The pressure tapped coreholder holds the core at an annulus pressure of 3000 psi. Each pressure transducer acquires the pressure data as a function of time. The outlet of the core is connected to a back pressure regulator which is set at 2300 psi. The back pressure regulator is connected to a liquid collecting burette and a wet test meter. High pressure (1/8 inch, O.D) stainless steel tubing is used to handle the flow in the system. The syringe pump delivers the desired fluid at constant rate to the coreholder. Transducers located at equal distances from each other measure the pressure drop across the core. Each of the transducers are calibrated against the standard pump pressure before starting an experiment. The signals are converted and transmitted to a computer for data recording. The fluids exit the core at a pressure greater than the back pressure and are collected in the collecting burette or passed through to the wet test meter. Oil and water collection is monitored using a camera interfaced with the computer for data storage.

There are at least four sets of experiments to be carried out: a continuous injection $\mathrm{CO}_{2}$ process, a Water Alternating Gas (WAG) process, a Micro-Dispersed Water Gas Mixture (Bortkevitch, et al, 2006) experiment (simultaneous injection of $\mathrm{CO}_{2}$ and water) and a surfactant enhanced $\mathrm{CO}_{2}$ flood (a foam flood with $\mathrm{CO}_{2}$ ). The continuous injection experiments have been completed and the WAG and water-gas mixture experiments are in progress. Each of these experiments has to undergo a sequence of cycles before exposure to the different tertiary flooding schemes. The sequence is (a) core cleaning to get rid of the fluids in the core; (b) flooding with brine to determine absolute permeability; (c) oil flooding to connate water saturation with determination of end point relative permeability; (d) water flooding to residual oil saturation to determine the end point relative permeability; and (e) the tertiary flooding process under consideration. It is believed that conducting these experiments on the same core system under nearly identical operating guidelines will allow this work to better evaluate the displacement mechanisms in question so that the information can be used in conjunction with scaling work to better design sweep improvement possibilities in both new and existing floods. 


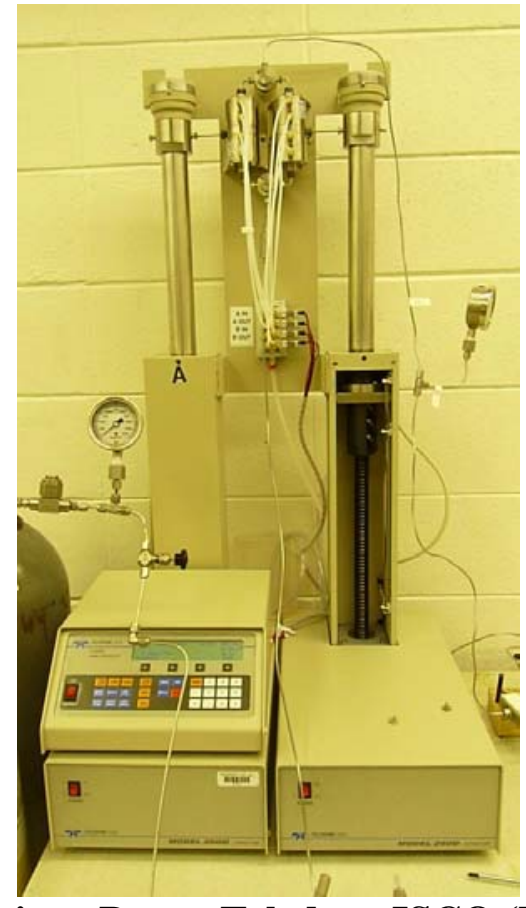

Figure 3: Syringe Pump-Teledyne ISCO (Model: 260D)

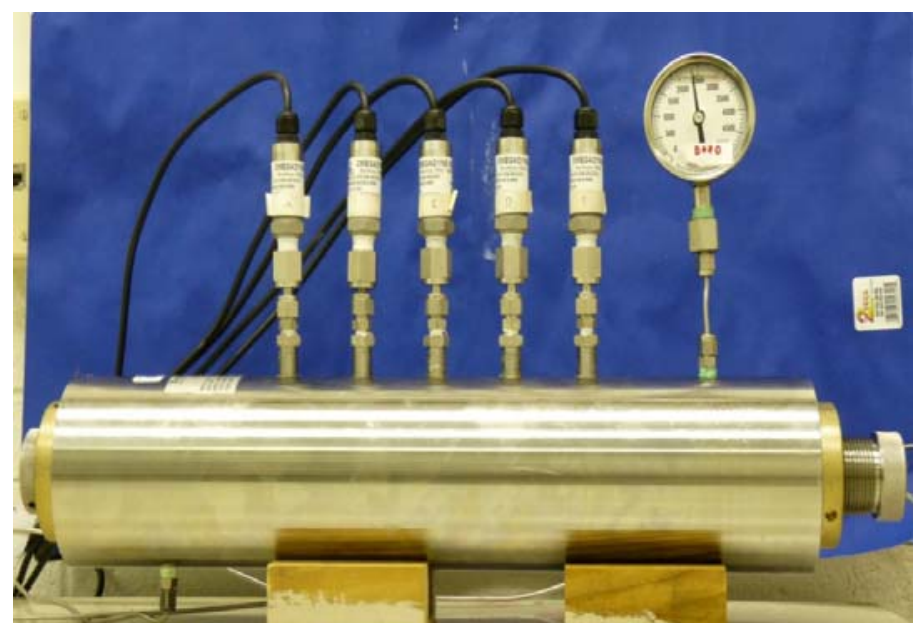

(A)

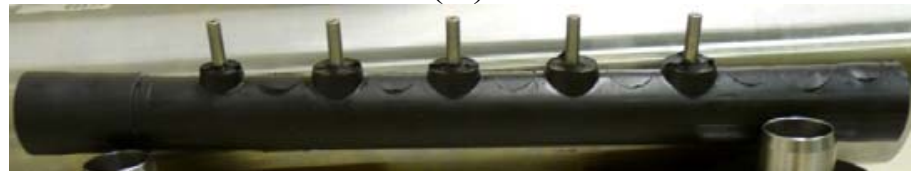

(B)

Figure 4: Pressure Tapped Hassler Type Core Holder (A) and VITON Sleeve (B) 


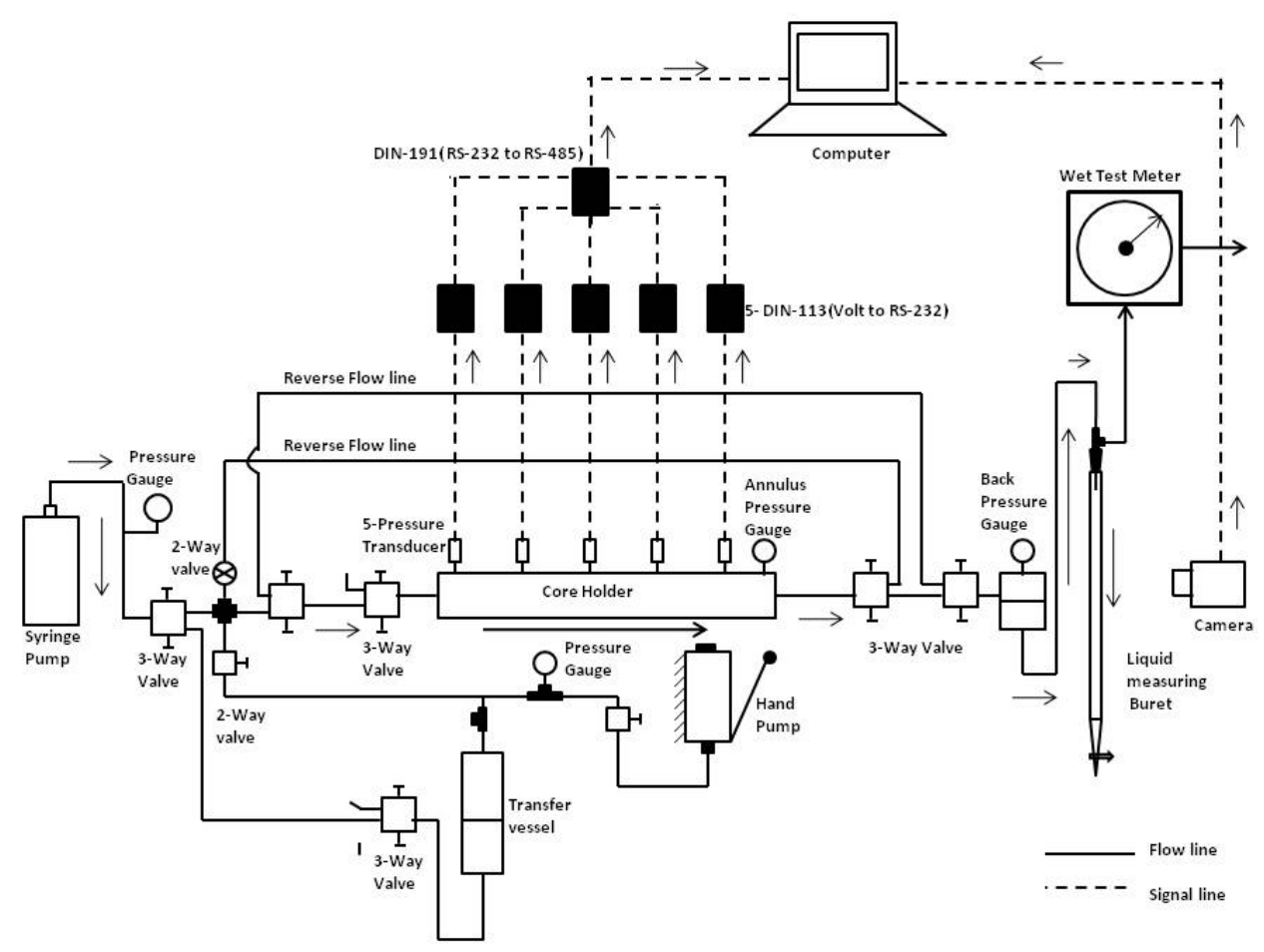

Figure 5: Schematic Horizontal Core flooding Apparatus

The porosity of the core was found to be $17.84 \%$ using the water weight method. The continuous injection process recovered $5.95 \mathrm{cc}$ of residual oil for a displacement efficiency of $94.65 \%$ while the WAG process recovered $7.0 \mathrm{cc}$ of residual oil and had a displacement efficiency of $91.90 \%$. Table 1 lists the experimental result for these tests.

Table 1: Experimental Results for Continuous Injection and 20\% Slug Size WAG

\begin{tabular}{|c|c|c|c|c|c|c|c|}
\hline $\begin{array}{c}\text { Experiment } \\
\text { Description }\end{array}$ & $\begin{array}{c}k \\
(\mathrm{mD})\end{array}$ & $S_{w c}$ & $\begin{array}{c}\text { Endpoint } \\
k_{r o}\end{array}$ & $S_{o r}$ & $\begin{array}{c}\text { Endpoint } \\
k_{r w}\end{array}$ & $\begin{array}{c}\text { Tot. RF } \\
\%\end{array}$ & $\begin{array}{c}\text { Util Fct } \\
(\mathrm{Mcf} / \mathrm{bbl})\end{array}$ \\
\hline Continuous Injection & 26.44 & 0.1929 & 0.7705 & 0.2674 & 0.1008 & 94.65 & 21.1 \\
\hline $20 \%$ slug size WAG & 25.08 & 0.2205 & 0.5413 & 0.3270 & 0.0821 & 91.90 & 13.1 \\
\hline
\end{tabular}

The next steps in this work will be to evaluate several additional WAG slug sizes and to test the simultaneous injection of $\mathrm{CO}_{2}$ and water process. This will be done using 4 ratios of gas to water. In addition, the effect of a surfactant solution on the $\mathrm{CO}_{2}$ displacement process will be evaluated. These experiments have been designed and preliminary work has been conducted to work with two surfactant vendors. The project team has also had conversations with Dr. Robert Enick at the University of Pittsburgh as has Denbury Resources personnel. Discussions to further develop an efficient process for evaluation have begun.

In addition, an understanding of how the results obtained in laboratory experiments apply to field-scale processes is ongoing. Dimensionless groups from both dimensional analysis and inspectional analysis are being developed and tested through simulation. The key characteristic 
of this work is that these groups need to be both robust mathematically and understood practically. Most of the groups have length dependent terms (for instance an area term) that when evaluating laboratory work make sense (the total cross-sectional area of the core) but when evaluating a field process would need to be adjusted. The appropriate cross-sectional area for an EOR process might be a portion of the zone you are trying to divert fluid away from or into. In addition to the theoretical and numerical work an experiment has been designed that will leverage the fact that the coreholder purchased for the project is identical to another one in use here at LSU. The experiment will test heterogeneous displacements by having the coreholders run in parallel with differing permeability cores. That work will commence once the simultaneous gas-water and the surfactant experiments have finished later this year.

\section{Subtask 1.2: Study of $\mathrm{CO}_{2}$ Conformance and Sweep Efficiency}

There were three main items associated with Subtask 1.2. First, historical reservoir, production and well data was to be used to evaluate the effectiveness of $\mathrm{CO}_{2}$ injection and sweep in the Little Creek Field. This item incorporated and extended tools developed by Albertoni (2002) and Dinh (2003) for waterflood evaluation to $\mathrm{CO}_{2}$ floods with some success. An alternative evaluation technique was proposed which allows the relationship between injector/producer pairs to be evaluated on a more statistical basis. Second, production logs acquired by Denbury Resources, Inc. as part of their cost sharing arrangement were used to evaluate injection profiles in two wells to identify vertical injection conformance and isolate injection issues. These logs were also incorporated into the Subtask 1.3 simulation study to help ground that study in reality. Third, a cased-hole logging program was developed and Denbury Resources, Inc. paid for the acquisition of the logs required to test whether the developed procedure could be used to estimate oil and/or $\mathrm{CO}_{2}$ saturation to identify potential unswept segments of the reservoir. Denbury not only acquired the logs to test the program on a well recommended by the investigators, they also ran an additional suite of cased-hole logs on a well that had old logs that are more common to their other operations. They did so both to fulfill their cost sharing obligation and to test the methodology on a much more difficult case but one that has the potential to either stimulate additional recovery from fields thought to be "gassed out" or to prevent unnecessary expenditures to test those same fields.

\section{Evaluation of Interwell Connectivity in $\mathrm{CO}_{2}$ Flooding}

For an effective reservoir development plan, a sound reservoir description and a better knowledge and understanding of how the field was and is being operated is essential. The acquisition and processing of some of this information throughout the life of the reservoir is expensive and, in many cases the information required is unavailable. The resources for building and using various modeling methods such as numerical simulation and the lack of important information make the process of reservoir characterization difficult.

In an enhanced oil recovery or secondary recovery system where the production rates of individual wells are affected by injection rates in that system, an understanding of the interwell communication would maximize the performance of an existing flood. Various production and recovery analysis methods have been used to better understand flood performance, but these techniques do not use the production and injection rate data to quantitatively determine injector- 
producer well pair connectivity. In recent years, the quest to quantitatively assess the relationship between injectors and producers to better understand sweep efficiency has seen increasing interest. Large sets of production and injection rate data of the various wells are required to evaluate the influence each injector has on each producer. Various statistical approaches have been used to ascertain the physical relationship between injector-producer well pairs in waterfloods. These methods are not nearly as costly as the sophisticated models that are typically used for reservoir engineering in the oil industry. The knowledge gained from these statistical approaches can yield improved operating practices to improve oil recovery in active floods, and to form strategies for implementing new floods.

Several methods have been developed to evaluate the rate performance of an existing well with that of the surrounding injectors. Ogunyomi (2009) presents a detailed report and evaluation of these techniques and determined that a method from Albertoni (2002) would be the tool that had the best chance at evaluating sweep efficiency in a $\mathrm{CO}_{2}$ flood.

Albertoni (2002) presented two statistical methods used to evaluate the connectivity between injectors and producers in a waterflood. These were called the Multivariate Linear Regression (MLR) and the Balanced Multivariate Linear Regression (BMLR) method. Albertoni (2002) views the reservoir as a system that processes a stimulus (injection) and returns a response (production). The reservoir effect on the input signal (injection) and the output signal (production) is dependent on the location and the orientation of each injector-producer pair in the system. This technique uses different statistical methods based on constrained multivariate linear regression to quantitatively determine the communication between wells in the system. Diffusivity filters were used to account for the time lag and attenuation that occurs as fluid flows between the injector and producer. Albertoni (2002) applied the methods to a synthetic field generated by numerical simulation with five-spot injection patterns and also to a waterflood in Argentina. Results were very difficult to validate, but seemed to agree with the presence of known geological features.

Dinh (2003) used these same techniques on a synthetic reservoir model using the BOAST98 numerical simulator and then to a waterflood field in northeastern Oklahoma. The results obtained by Dinh (2003) from the numerical models reflected the characteristics of anisotropy, vertical heterogeneity, sealing faults and flow channels. Dinh (2003) concluded that for a media with small dissipation, a short diffusivity filter function should be considered and further analysis to determine how frequently the flowrates being used in these types of models should be measured.

Albertoni (2002) presented the MLR method to estimate the production rate of a producer well $j$, as

$\hat{q}_{j}(t)=\beta_{o j}+\sum_{i=1}^{I} \beta_{i j} i_{i}(t) \quad(j=1,2, \ldots N)$

where $N$ is the total number of producers and $I$ is the total number of injectors. This equation states that for any given time period, the total rate of a production well is a linear combination of the rates of every injector in the field plus a constant $\beta_{o j}$ term. The $\beta_{o j}$ term is a constant that tries to account for the unbalance in the field. This unbalance will include liquid production not associated with injected fluid (primary production), as well as injection losses (injection not affecting producers). Using field data, Equation 1 suggests that injection rate changes in the model cause instantaneous production rate changes which would imply steady state flow in the reservoir. In many cases, there are time lags and attenuation that occur as compressible fluids 
flow from injectors to producers. Diffusivity filters were proposed to account for the time lag and attenuation in the system. The $\beta_{i j}$ terms are the weighting coefficients that represent the proportion of the production rate attributed to each injector or the "connectivity" between the injectors and the producer. Because the model assumes that no linear relationship exists between active injectors in the system, injector rate variations should only influence the production rates values in the system and not the other injection rates. For fields where the injection and production rates are balanced (total injection and production rates are equal), this assumption seems reasonable. For unbalanced systems, this assumption is questionable. Once all the parameters are determined, the model quantifies how each injector influences each producer.

Results obtained by both Albertoni (2002) and Dinh (2003) indicated that very small negative weighting coefficients existed between well pairs on opposite sides of faults or barriers. Albertoni (2002) stated that these negative coefficients have no physical interpretation and should be considered as zeros, indicating that no communication exists between the well pairs.

Albertoni (2002) introduced an approach for eliminating the negative weighting coefficients called the successive elimination of negative weighting coefficients (SEN). First, the most negative $\beta_{i j}$ weighting coefficient is set to zero which eliminates that well pair from consideration. Next, the regression method is performed again recalculating the entire set of weighting coefficients with one fewer injector-producer well pairs. If there are additional negative weighting coefficients, the new most negative weighting coefficient is set to zero, and the $\beta_{i j}$ weighting coefficients are again recalculated. This procedure is repeated until no negative coefficients remain. The SEN procedure was extended to also eliminate large positive weighting coefficients (those greater than 1). This process is called the successive elimination of physically non-significant weighting coefficients (SEP). Application of the SEN and SEP procedure to the MLR model would therefore eliminate the well pairs with the largest influence in the model and account only for the well pairs with lower influence.

One of the assumptions of the MLR models is that the injection and production conditions are constant. Periods where the producers are shut-in should be excluded from the analysis. Therefore a portion of the Little Creek Field needed to be analyzed since there are few time periods where injection and production at all wells were continuous. The area analyzed was a portion of the field (called Phase 2) that included 11 producers and 4 injectors between January 1, 1989 and December 31, 1991. The total injection and production values for the system during this period were $27,471,739 \mathrm{RB}$ and $21,901,196 \mathrm{RB}$ respectively. The cumulative injection to withdrawal ratio (IWR) was 1.25 .

The MLR approach was applied to the data in this region both with and without diffusivity filters and with and without the use of the SEN and SEP techniques. Weighting coefficients calculated were both positive and negative. Generally, closer well pairs would be expected to have larger coefficients than well pairs that are further apart. Several of the largest values for the weighting coefficients were at the lower values for the separation distance. However, there were also a number of very low coefficient values at the smaller well pair distances as well and most of the negative values were in the middle to large distance values. After the application of the SEN and SEP procedure 13 negative weighting coefficients were eliminated and 6 positive weighting coefficients were eliminated. Out of the 44 injector-producer well pair coefficients in the original model, only 25 were accounted for with this new model. Only two of these 25 coefficients increased, while 9 remained the same and the remaining 14 decreased in magnitude. 
Albertoni (2002) and Dinh (2003) both used 6 and 12 months diffusivity filters in their work. Albertoni recommended the 12 month filter while Dinh recommended that no filter be used in his work. The actual time it takes for the production rate value of a producer to respond to an injection rate change of an injector should be the one applied to the MLR model. A radiusof-investigation-type calculation can be used to estimate the time it takes for the production rate at a producer to respond to an injection rate change at a particular injector. This time takes the form (Lee, 1982)

$$
t=\frac{948 \phi \mu c_{t} r_{i}^{2}}{k}
$$

In analyzing data from Little Creek, all but one of the injector-producer pairs had less than 1 month calculated times. This suggests that this is a low dissipation system and no more than a 1 month diffusivity filter should be applied.

For the case with one month diffusivity filters, results showed that there were both positive and negative linear relationships between the injector-producer well pairs. There was a slight decrease in the number of negative weighting coefficients between injector producer well pairs from 15 (for the case without diffusivity filters) to 14. Also, the magnitude of weighting coefficients generally increased as did the $R^{2}$ values of all but two producers with two remaining constant.

A comparison between the total modeled liquid production rate and the total observed liquid production rate for the standard MLR case showed a coefficient of determination $\left(R^{2}\right)$ value of 0.83 which would imply that the model results have not perfectly captured what is going on in the reservoir but the correlation is reasonably good. These results were lower than those seen in Albertoni (2002) and Dinh (2003) for their field cases. After the application of the SEN and SEP procedure the $R^{2}$ value decreased to 0.67 . However, the $R^{2}$ value increased from 0.83 to 0.86 after the application of the 1-month diffusivity filter.

Rose diagrams were used to show the magnitude and directionality of fluid flow suggested by the weighting coefficients. Interpreted fluid flow in the reservoir varied significantly depending on whether the unrestricted weighting coefficient method was used or whether the SEN and SEP procedures were used. Considering that all but two of the producers and all of the injectors show high flow rates with reasonably good pressure support throughout the area, the unrestricted weighting coefficient method appears to provide results which are consistent with the present understanding of the reservoir where all of the injectors broadcast their fluid out with few interpreted barriers or boundaries. Details of these results can be seen in Ogunyomi (2009).

Ogunyomi (2009) proposed that a Simple Linear Model (SLM) may be just as capable of evaluating the interwell connectivity as the MLR approaches. The SLM approach would be a somewhat less complicated method in comparison to the MLR approach and the significance of the relationship between each injector-producer well pair could be determined by a statistical significance hypothesis test.

The simple linear model for a production well $j$ is (Edwards, 1984) $q_{j}=a+b i_{i}$

where the liquid production rate of a well is now a linear function of the rate being injected at an injector.

The application of the simple linear model to the 11 producers and 4 injectors in the Little Creek study area indicated negative relationships and positive coefficients greater than 1 between 
some of the injectors and producers. However, in comparison to the MLR case, which had 15 negative weighting coefficients, only 2 negative weighting coefficients were obtained with the application of the SLM method. The significance of the relationships between each injectorproducer well pair was determined by a statistical significance hypothesis test. Both of the negative weighting coefficients were found to be statistically insignificant and could be ignored. However, similar to the cases with and without diffusivity filters, there were some well pairs with weighting coefficients greater than 1 that were deemed statistically significant.

Once the injector-producer well pairs with significant relationships were determined, the MLR approach was used to determine inter-well connectivity between injector-producer well pairs. In general, evaluation of the movement of fluids in the reservoir from the results of the MLR model without diffusivity filters, the MLR approach with one-month diffusivity filters and the MLR approach with significant injector-producer well pairs seem to be similar. Even after the elimination of the nonsignificant injector-producer well pairs, the results from the MLR model suggest that there are still 7 negative weighting coefficients. However, of the 7 negative coefficients only 3 are larger than -0.06 . The original MLR technique had 8 negative coefficients larger than -0.06 . This significant reduction in the number of large negative coefficients is encouraging in that small negative coefficients may be indicators of barriers (as shown by both Albertoni (2002) and Dinh (2003)), but there is no clear physical explanation for large negative coefficients. It was noted that many of the negative weighting coefficients seen might be interpreted as being fluid barriers as they were generally seen when evaluating the relationship between an injector/producer well pair that had another injector in between the well pair. This suggests that it may be possible to use the combination of the SLM and MLR methods to obtain some physical understanding of fluid movement.

Extension of this work has begun. A PhD student has investigated the work by Yousef (2006) as well as the work by Dinh and Tiab (2008) both of which depend on knowledge of the well bottomhole pressures. The proposed extension is to perform a very simple simulation of a reservoir using unstructured grids such that each well pair can be represented by a single value of connectivity between the well pairs. The resulting "history match" should result in constraining the well to well connectivity values and continues to require only injection and production data, but may need reasonable starting values for estimated reservoir pressure and transmissivity or permeability values.

\section{Production Logging in $\mathrm{CO}_{2}$ Flooding}

As shown above, historical reservoir, production and well data can be used to evaluate the effectiveness of $\mathrm{CO}_{2}$ injection and areal sweep in the Little Creek Field. Production logs are required to evaluate injection profiles in individual wells and to identify vertical injection conformance (vertical sweep) or isolate injection issues. As part of their cost-share, Denbury Resources, Inc. acquired three production logs in the active area of the Little Creek Field both to aid in the evaluation of the vertical sweep and also to help in evaluating the performance of the project reservoir simulation studies. One of the production logs could not be used due to problems with fill. A log from LCU 10-9 is shown in Figure 6 where an estimated 78\% of the fluid appears to be exiting the bottom 11 feet of the 46 foot zone. The actual log from this well is shown in Figure 7. Similarly, Figure 8 shows the header for the production log for LCU 23-9. An estimated $71 \%$ of the fluid appears to be exiting the bottom 2 feet of the 29 foot zone. 


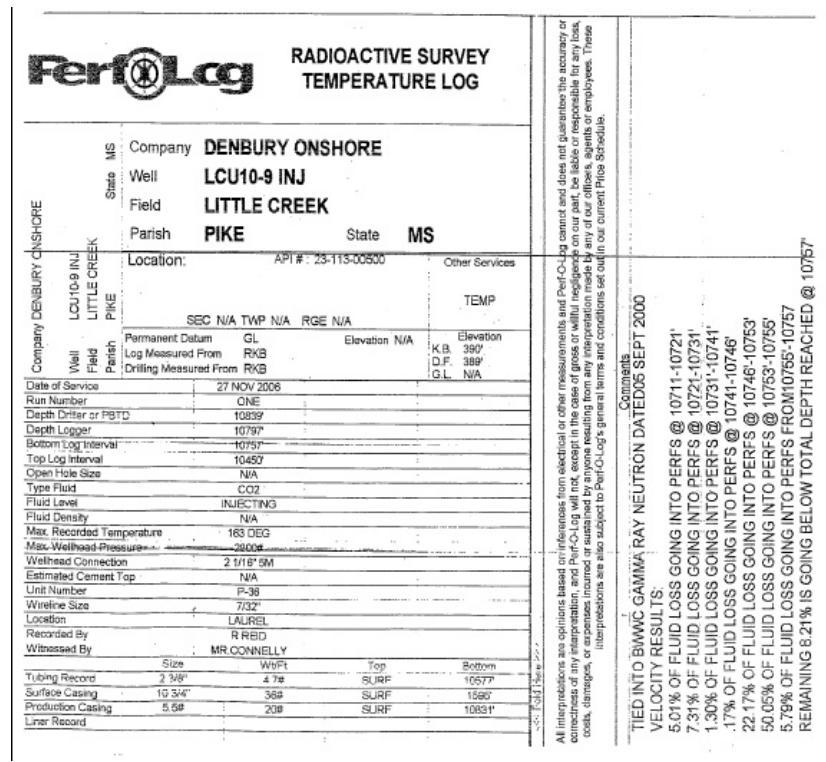

Figure 6: Header from Production Log for Little Creek Unit (LCU) 10-9. Note that an estimated $78 \%$ of the fluid appears to be exiting the bottom 11 feet of the 46 foot zone.
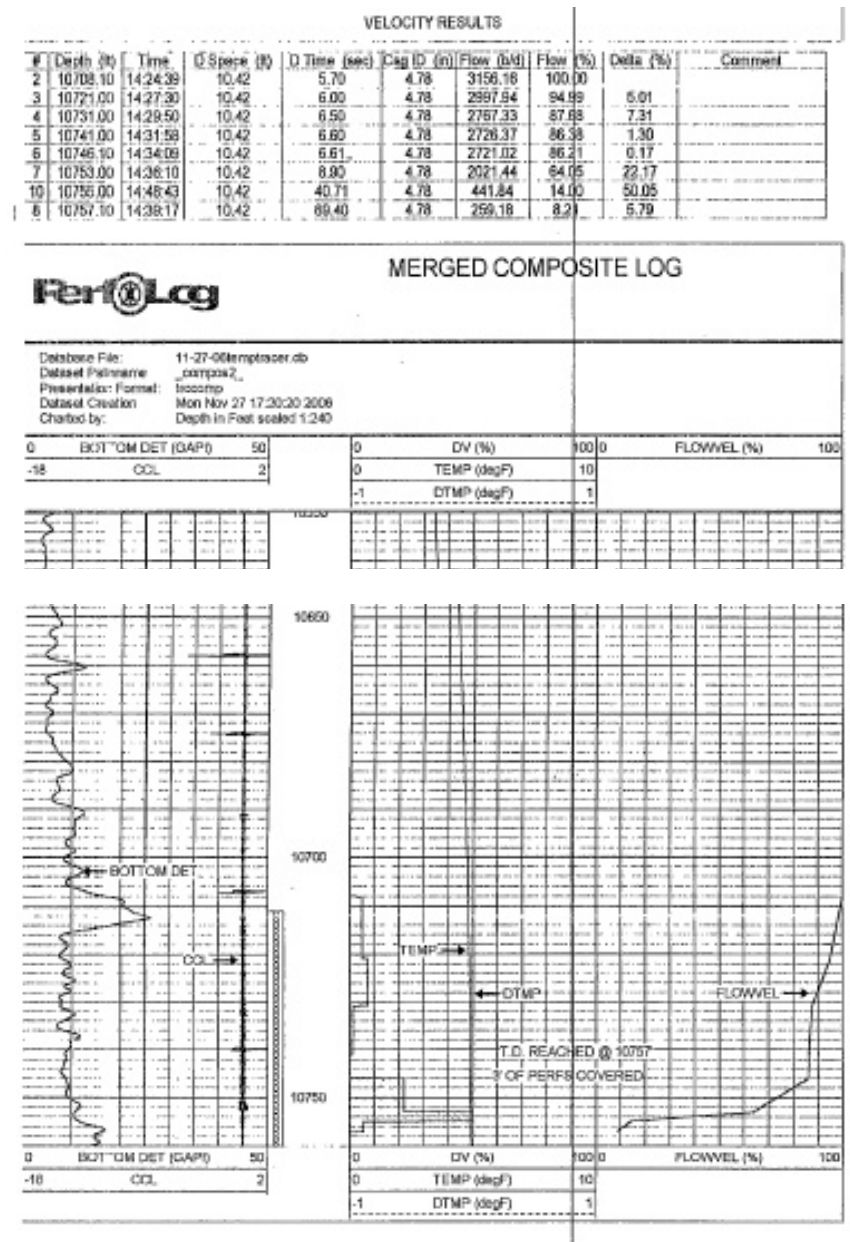

Figure 7: Production Log run from LCU 10-9. 


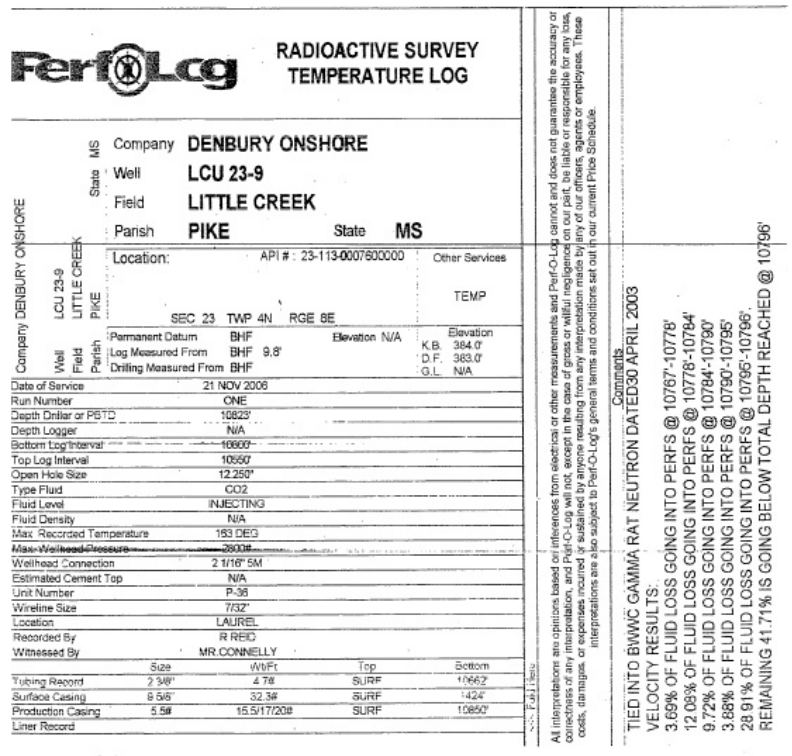

Figure 8: Header from Production Log for LCU 23-9. Note that an estimated $71 \%$ of the fluid appears to be exiting the bottom 2 feet of the 29 foot zone.

As Little Creek was generally thought to be a fairly homogeneous reservoir where gravity was the dominant mechanism controlling the $\mathrm{CO}_{2}$ flood, these results show that vertical sweep may not be controlled as much by gravity as once thought. If most of the displacing fluid is entering the reservoir in certain zones, sweep may be controlled by the speed at which the fluid moves through those zones. Simulation studies (see Subtask 1.3) seem to confirm this and techniques are being evaluated to improve these injection profiles.

\section{Cased-Hole Saturation Logging}

In this work, research efforts focused on methods to estimate the saturation profile in individual wells. To evaluate the amount of bypassed oil in a miscible $\mathrm{CO}_{2}$ flood, it is necessary to obtain some estimate of the oil saturation that remains in the reservoir. This determination calls for a tool or a sequence of tools that will distinguish oil from the other phases that may be present in situ, even if those phases are miscible with the oil. Conventional saturation tools are designed mainly for two or three immiscible phases, and may not be appropriate for a two or three phase miscible systems. This study evaluated different tools that might be used to estimate the oil saturation with a goal of determining the best tool or combination of tools to use in an existing reservoir that is undergoing a $\mathrm{CO}_{2}$ flood.

The study found that the combined use of two neutron tools could be used to evaluate the remaining oil saturation in a miscible $\mathrm{CO}_{2}$ flood. The techniques presented are not time lapse techniques and so are applicable even where there are no base cased-hole logs to evaluate. These methods are applicable in areas where $\mathrm{CO}_{2}$ has been injected for a long period of time without a base pre- $\mathrm{CO}_{2} \log$. The techniques work best with a full suite of open-hole logs to characterize the petrophysical properties of the well. However, if petrophysical properties are known or reasonably estimated from other available data, the proposed methods can provide an estimate to the oil saturation that should be adequate to allow go/no go decisions. 
Cased-hole logging tools are currently being used to evaluate two-phase (oil/water) systems and some $\mathrm{CO}_{2}$ systems but only in a time-lapse sequence. One neutron tool (Svor and Globe, 1984) has a long- and short-spaced detector and has been proposed for use in miscible systems, but it is unclear as to whether this is being done successfully.

In miscible $\mathrm{CO}_{2}$ systems, the $\mathrm{CO}_{2}$ is usually in a supercritical state and has density characteristics that are closer to those of oil than those of gas. This characteristic makes it difficult for tools that differentiate gas from liquid. Unlike oil-water systems where carbon is seen as the characteristic element for oil and oxygen is the characteristic element for water, the introduction of $\mathrm{CO}_{2}$ in a miscible state with the oil, makes it difficult for tools that use this distinguishing characteristic to measure oil saturation. The oxygen in the $\mathrm{CO}_{2}$-oil mixture is interpreted as if it is from water and the carbon as if it is only from the oil.

To overcome this difficulty Amadi and Hughes (2008) discussed the advantages and disadvantages of different cased-hole saturation logging tools and found that a combination of neutron tools should be able to provide an estimate of saturation values in a three-phase system. The three neutron tools reviewed were the epithermal neutron, the pulsed neutron and the carbon-oxygen log.

The carbon-oxygen tool emits periodic bursts of high energy (14 million electron volts) neutrons. These high energy (fast) neutrons bombard the nuclei of the material and impart some of their energy to the target nuclei, thereby slowing down their speed and exciting the nuclei of the material to a higher unstable bound-state. The excited state lasts less than a microsecond, and de-excitation returns the nucleus to its ground state by prompt emission of one or more gamma rays at energies unique to the target nucleus. A reaction involving carbon ${ }^{12} \mathrm{C}$ has an excitation half-life of $3.8 \times 10^{-14} \mathrm{~s}$, and a gamma ray of energy $4.44 \mathrm{MeV}$ is produced. For oxygen, ${ }^{16} \mathrm{O}$ the principal emitted gamma-ray energy is $6.13 \mathrm{MeV}$ and has an excitation half-life of $1.7 \times 10^{-11} \mathrm{~s}$. The oxygen spectrum contains other inelastic peaks, at 6.92 and $7.12 \mathrm{MeV}$ (Serra, 2004). Prompt gamma-ray measurement from inelastic scattering is indicative of the relative concentration of carbon and oxygen, and these relative concentrations are used to evaluate oil saturation.

The pulsed neutron tool emits pulses of high-energy neutrons. Following each pulse, at about 1000 microseconds or longer, the neutrons are rapidly slowed down in the formation to low energy "thermal" neutrons. Upon collision with the nuclei of the formation, the thermal neutrons are captured with corresponding emission of gamma rays (Youmans, et al, 1964). The rate of thermal neutron capture is characteristic of the capturing nuclei, and depends on the mean thermal velocity, $v$, (a constant for a given temperature) and the macroscopic capture crosssection of the medium. The measure of the probability of the capture is the capture cross section (symbol 2 ) in capture units (c.u). Chlorine has the highest capture cross-section among common earth elements, and therefore thermal neutron tools are chlorine sensitive. Fresh water has a capture cross-section of $22 \mathrm{c} . \mathrm{u}$ at $75^{\circ} \mathrm{F}$, while oil, with a GOR less than 10,000 scf/stb, has a capture cross section between 20 and 22 c.u. This closeness in capture cross section between fresh water and oil makes it difficult to differentiate oil from fresh water. As a rule of thumb, a minimum of 15 percent porosity and 50,000 ppm equivalent $\mathrm{NaCl}$ formation water salinity are required for quantitative evaluation (Smolen, 1996).

The epithermal neutron logging tool was originally designed for use in porosity determination. For this reason, it is also called the Neutron Porosity Tool. The tool operates at the epithermal neutron energy level. At this energy level, the neutron interacts with the formation elastically, and the neutrons are rapidly slowed down by elastic collision with nuclei. The neutrons collide with nuclei in a manner similar to that of two colliding billiard balls, and the 
energy lost per encounter is a function of the incident angle of the collision, and the mass of the target nucleus. The energy lost by the incident neutron is transferred to the nucleus in the form of kinetic energy. Therefore the internal energy of the nucleus remains unchanged and no excitation and no radiation are associated with elastic scattering (Bassiouni, 1994).

Based on the mechanics of elastic collisions, the maximum energy will be lost when the target nucleus has a mass equal to that of the incident neutron. Therefore hydrogen atoms most strongly affect the neutron slowdown. This is because the single proton of the nucleus has approximately the same mass as a neutron. The probability of a collision occurring with a particular element is a function of the atomic concentration of the element per unit volume of the formation, and the elastic interaction cross section.

Since the oil-water- $\mathrm{CO}_{2}$ problem is really a compositional problem where the relative contributions of carbon and oxygen for the "oil", water and $\mathrm{CO}_{2}$ will change with time, this approach likely requires an iterative effort to try to evaluate these displacement effects. A simpler approach would be to assume that the "oil", water and $\mathrm{CO}_{2}$ all track as their own pseudocomponents with no mixing. This approach would be similar to black-oil modeling of solvent flooding where oil, water, gas and solvent are all tracked as being in their own "phase". These are really pseudo-components, but similar to simulation we will use saturation symbols in our notation. In reservoir simulation, a mixing parameter can be used to simulate the mixing that takes place amongst the fluids. Here we assume that there is no mixing.

For all of the neutron tools, the response to the tool can be viewed as a linear combination of the response from the formation and the borehole fluids. Amadi and Hughes (2008) showed that the oil saturation in an oil-water- $\mathrm{CO}_{2}$ system can be obtained from a carbon-oxygen $\log$ by

$$
S_{o}=\frac{c\left[\gamma_{m a}\left(1-\phi-V_{S h}\right)+\gamma_{S h} V_{S h}+\delta \phi+B_{o}\right]-A \alpha_{m a}\left(1-\phi-V_{S h}\right)-A \alpha_{S h} V_{S h}-A B_{c}-\phi S_{C O_{2}}[c / \delta(\delta-\omega)+A \eta]}{\phi(\beta+\% \delta)}
$$

where, $A$ is the relative nuclear cross sections of carbon and oxygen, $\alpha$ is the atomic density of carbon in the rock (with subscripts ma for matrix and Sh for shale), $\omega$ is the atomic density of oxygen in the rock, $\beta$ is the atomic density of carbon in the oil pseudo-component, $\delta$ is the atomic density of oxygen in the water, $\omega$ is the atomic density of oxygen in the $\mathrm{CO}_{2}$ pseudocomponent, $\eta$ is the atomic density of carbon in the $\mathrm{CO}_{2}$ pseudo-component $\left(\eta\right.$ is $\rho_{\mathrm{CO} 2} / 44$ and $\omega$ is $\left.\rho_{\mathrm{CO} 2} / 22\right), B_{c}$ is the carbon contribution from the borehole, and $B_{o}$ is the oxygen contribution from the borehole.

In sandstone rocks and water filled boreholes, both the matrix carbon and borehole carbon terms drop out $\left(\alpha_{m a}=0\right.$, and $\left.B_{c}=0\right)$. In addition, most shales have no carbon component so $\alpha_{m a}$ is also 0. Equation (4) resorts to a solution North (1987) presented for oil-water systems $\left(S_{\mathrm{CO} 2}=0\right)$. Presuming there is an independent evaluation of the porosity and that the atomic weight terms can be adequately evaluated, there are still two unknowns in Equation (4). Thus another tool needs to be used to obtain a solution for this problem.

Note that knowledge of the formation is very important since limestone $\left(\mathrm{CaCO}_{3}\right)$ and dolomite $\left(\mathrm{CaMg}\left(\mathrm{CO}_{3}\right)_{2}\right)$ contribute both carbon and oxygen while sandstone $\left(\mathrm{SiO}_{2}\right)$ contributes no carbon. Shaley sands with elemental carbon or oxygen will also cause difficulties. For twophase systems, fan charts can be generated to evaluate how the ratio of carbon to oxygen varies with porosity, fluid type and saturation and the makeup of the matrix.

Similarly, Amadi and Hughes (2008) showed that the oil saturation can be obtained from the pulsed neutron response for an oil-water- $\mathrm{CO}_{2}$ system from 


$$
S_{o}=\frac{\Sigma_{\log }-\left(1-\phi-V_{s h}\right) \Sigma_{m a}-V_{s h} \Sigma_{s h}-\phi \Sigma_{w}+\phi S_{C O_{2}}\left(\Sigma_{w}-\Sigma_{C O_{2}}\right)}{\phi\left(\Sigma_{o}-\Sigma_{w}\right)}
$$

where, $\Sigma_{\log }$ is the bulk capture cross section of the formation, $\Sigma_{o}$ is the oil capture cross section, $\Sigma_{w}$ is the capture cross section of water, and $\Sigma_{m a}$ is the capture cross section of the matrix. The volume fraction of the rock matrix includes everything which is not shale; therefore $\Sigma_{\text {ma }}$ represents not only the main rock minerals but also their impurities and cementing material. The $\Sigma_{\text {sh }}$ term can be determined in each case by measuring the average $\Sigma$ value recorded in shales surrounding the formation under study. The $\Sigma_{0}$ term can also be obtained, when the gas-oil ratio is known from correlations provided by the logging companies. The $\Sigma_{w}$ term can be obtained if water analysis is available or again using correlations, charts or $\Sigma-\phi$ cross plots (Schlumberger, 1972). The $\Sigma_{\mathrm{CO} 2}$ term can be calculated as

$$
\Sigma_{\mathrm{CO}_{2}}=0.05394 \rho_{\mathrm{CO}_{2}}
$$

For the epithermal neutron system, either a hydrogen index or a retardation index can be used as shown by Amadi and Hughes (2008). Using the retardation index form and solving for oil saturation yields

$$
S_{o}=\frac{\phi_{N}-\left(1-\phi-V_{s h}\right) R I_{m a}-V_{s h} R I_{s h}-\phi R I_{w}+\phi S_{C O_{2}}\left(R I_{w}-R I_{C O_{2}}\right)}{\phi\left(R I_{o}-R I_{w}\right)}
$$

The technique that was proposed was based on the assumption that the petrophysical properties are constant throughout the life of the reservoir, that there is no free hydrocarbon gas present and that the oil, water and solvent can be tracked as separate constituents. As previously shown, there are three tools that can be used to solve for the two unknowns in the oil saturation equations. Any two of the equations developed can be used to solve for the oil saturation. The simultaneous solutions for oil saturation all have the same form

$$
S_{o}=\frac{b_{1} a_{2}-b_{2} a_{1}}{a_{2}-b_{2}}
$$

For the epithermal neutron-pulsed neutron combination

$$
\begin{array}{lr}
a_{1}=\frac{\phi_{N}-\left(1-\phi-V_{S h}\right) \gamma_{m a}-V_{S h} \gamma_{S h}-\phi \gamma_{w}}{\phi\left(\gamma_{o}-\gamma_{w}\right)} & a_{2}=\frac{\left(\gamma_{w}-\gamma_{C O_{2}}\right)}{\left(\gamma_{o}-\gamma_{w}\right)} \\
b_{1}=\frac{\Sigma_{\log }-\left(1-\phi-V_{S h}\right) \Sigma_{m a}-V_{S h} \Sigma_{s h}-\phi \Sigma_{w}}{\phi\left(\Sigma_{o}-\Sigma_{w}\right)} & b_{2}=\frac{\left(\Sigma_{w}-\Sigma_{C O_{2}}\right)}{\left(\Sigma_{o}-\Sigma_{w}\right)}
\end{array}
$$

The solution for oil saturation then is

$$
S_{o}=\frac{\left.\left(\gamma_{w}-\gamma_{C O_{2}}\right)\left(\Sigma_{\log }-\left(1-\phi-V_{S h}\right) \Sigma_{m a}-V_{S h} \Sigma_{s h}-\phi \Sigma_{w}\right]-\left(\Sigma_{w}-\Sigma_{C O_{2}}\right) \mid \phi_{\log }-\left(1-\phi-V_{S h}\right) \gamma_{m a}-V_{S h} \gamma_{S h}-\phi \gamma_{w}\right\rfloor}{\phi\left[\left(\Sigma_{w}-\Sigma_{C O_{2}}\right)\left(\gamma_{w}-\gamma_{o}\right)-\left(\Sigma_{w}-\Sigma_{o}\right)\left(\gamma_{w}-\gamma_{C O_{2}}\right)\right]}
$$

For the carbon-oxygen and pulsed neutron combination the terms are slightly more complicated due to the possible carbon and oxygen responses in the rock. The terms are

$$
\begin{array}{ll}
\left.a_{1}=\frac{C\left[\gamma_{m a}\left(1-\phi-V_{S h}\right)+\gamma_{S h} V_{S h}+\delta \phi+B_{o}\right]-A \alpha_{m a}\left(1-\phi-V_{S h}\right)-A \alpha_{S h} V_{S h}-A B_{c}}{\phi(\beta+C / \delta} \delta\right) & a_{2}=\frac{-(\%(\delta-\omega)+A \eta)}{(\beta+C / \delta)} \\
b_{1}=-\frac{\Sigma_{\log }-\left(1-\phi-V_{S h}\right) \Sigma_{m a}-V_{S h} \Sigma_{s h}-\phi \Sigma_{w}}{\phi\left(\Sigma_{w}-\Sigma_{o}\right)} & b_{2}=-\frac{\left(\Sigma_{w}-\Sigma_{C O_{2}}\right)}{\left(\Sigma_{w}-\Sigma_{o}\right)}
\end{array}
$$

Solving for oil saturation then yields 


$$
\begin{aligned}
& S_{o}=\frac{(C(\delta-\omega)+A \eta)\left[\Sigma_{\log }-\left(1-\phi-V_{S h}\right) \Sigma_{m a}-V_{S h} \Sigma_{s h}-\phi \Sigma_{w}\right\rfloor}{\phi\left[\left(\Sigma_{w}-\Sigma_{C O_{2}}\right)(\beta A+C / o)-\left(\Sigma_{w}-\Sigma_{o}\right)(\%(\delta-\omega)+A \eta)\right]} \\
& +\frac{\left(\Sigma_{w}-\Sigma_{\mathrm{CO}_{2}}\right)\left[\%\left[\gamma_{m a}\left(1-\phi-V_{S h}\right)+\gamma_{S h} V_{S h}+\delta \phi+B_{o}\right]-A \alpha_{m a}\left(1-\phi-V_{S h}\right)-A \alpha_{S h} V_{S h}-A B_{c}\right]}{\phi\left[\left(\Sigma_{w}-\Sigma_{\mathrm{CO}_{2}}\right)(\beta A+C / \delta)-\left(\Sigma_{w}-\Sigma_{o}\right)(\%(\delta-\omega)+A \eta)\right]}
\end{aligned}
$$

Amadi and Hughes (2008) showed epithermal neutron/pulsed neutron and pulsed neutron/carbon-oxygen cross plots as a function of porosity and showed the application of the process to several synthetic cases. These synthetic cases showed that there is a strong response to all of the tools when the logs are run in time-lapse mode. The responses are much more subtle when there is no previous log to compare to, but there was a signal to detect if one was careful.

Since the epithermal neutron tool is most strongly influenced by hydrogen interactions and since $\mathrm{CO}_{2}$ is effectively displacing hydrogenated fluids, it was felt that this tool would be a key to finding where the $\mathrm{CO}_{2}$ has swept. We believe that the epithermal neutron-pulsed neutron combination may be a superior combination to try as it possibly has less uncertainty. Note that the $\mathrm{C} / \mathrm{O} \log$ response effects nearly all parts of the $\mathrm{C} / \mathrm{O}$-pulsed neutron saturation equation seen in Equation 12.

Most, if not all of the logging companies have epithermal tools that are too large to be run inside tubing and thus require a workover to be performed in order to run such an evaluation. The most common combination of tools to run then is likely the pulsed neutron and carbonoxygen combination. The $\mathrm{C} / \mathrm{O}$ and pulsed neutron logs being run today also measure much more than the primary parameters developed for the technique presented in this work. There is likely considerable ongoing research at the logging company research centers as to how to make these tools highly accurate for flood monitoring especially for the time-lapse techniques (see Müller, et al, 2007 for a $\mathrm{CO}_{2}$ Sequestration example).

Our purpose is in this work has been slightly different in that our efforts are focused on those cases where an operator has been flooding for an extended period of time but did not run a base $\log$ prior to initiating the flood. They may run another log in the future, but would like to have an idea as to whether there is oil being bypassed near their injectors or producers. Under these circumstances about the best you can hope for as far as data availability are open-hole logs, core analyses and fluid samples.

A recommendation was made to Denbury Resources, Inc. to run the pulsed neutron/carbon-oxygen combination in an existing well. Several available wells in DRI fields were evaluated to determine a suitable well to test the methodology. The first well recommended by the project team was one of the largest producers in a particular field and so was rejected. That recommendation was made due the fact that the well was a recently drilled well which had both modern open hole logs and a core evaluation. A petrophysical study tying the core to the open hole logs had also been done prior to the logging study. To still leverage this quality information, a well nearby in the same field with high quality modern logs was selected as the first test case. Since the majority of wells DRI operates do not have complete suites of modern open hole logs, they wanted to see what the evaluation technique would find in a well with a more typical data set for these wells. Thus, an additional well in the same field was recommended where all of the open hole logs were run in the mid-1960s. Denbury Resources paid for these logs as part of their cost-share amount and the result was that their total cost share was 19\% higher than their original commitment.

The well with the higher quality data was evaluated based on the tie to the core information. This well was completed in a different zone than the target zone so should be 
similar to logging a monitor well in a flood. The logs evaluated using the techniques developed in this work show distinct zones of predominantly $\mathrm{CO}_{2}$ in 2 to 10 foot zones which were interpreted to be reasonable representations of what was believed to be happening in the reservoir (see Figure 9). As this well was a monitor well in the zone of interest the results could not be directly tested but were deemed reasonable by Denbury Resources personnel. Processing the log also showed that there were portions of the log where carbon-oxygen ratio values were negative. This unusual occurrence is still being investigated.

Results from the well with lower data quality were both much more difficult to obtain and highly uncertain. Because there were no open hole porosity logs available, porosity values needed to be obtained from the cased hole log in addition to the fluid content. Shale content also was uncertain due to a different way of obtaining shale content required by the older logs. In the direct ties to the core, the recommendation was to determine shale content from gamma ray logs. The quality of the gamma ray logs for this well were poor and the gamma ray signature had changed considerably from the original open hole logs to the cased hole log. Neither the open hole gamma ray nor the cased hole gamma ray then were deemed trustworthy for computing shale content. SP logs were chosen as the tool to compute shale content and they were generally smoother and reflect gross sand-shale content more so than the modern open hole gamma ray logs making the computed shale content values uncertain. Figure 10 shows the results from the analysis and while the results appear reasonable, the uncertainty in the calculation method is only partially reflected in that the method was much less consistent than was obtained in the well with higher data quality. However, again qualitative evaluation of $\mathrm{CO}_{2}$ locations within the wellbore was deemed reasonable.

The general conclusions from this work were that certainly time lapse monitoring of $\mathrm{CO}_{2}$ floods is something that should be reasonably accurate. A considerable amount of effort in preliminary evaluation of petrophysical properties as well as post-processing of the combination logs are required but the combination logs can provide insight into the current saturation state in a well without the requirement for time lapse techniques. In addition, the operator has become convinced that baseline cased hole monitor logs are a necessity in their new floods. Once a reasonable methodology to process cased hole logs for cases where data quality is less than optimal is accomplished, it is likely that they will also begin a process of identifying areas they believe require further investigation where diagnosing oil bypassing and poor sweep may instigate mediation techniques to improve recovery and/or $\mathrm{CO}_{2}$ utilization. 


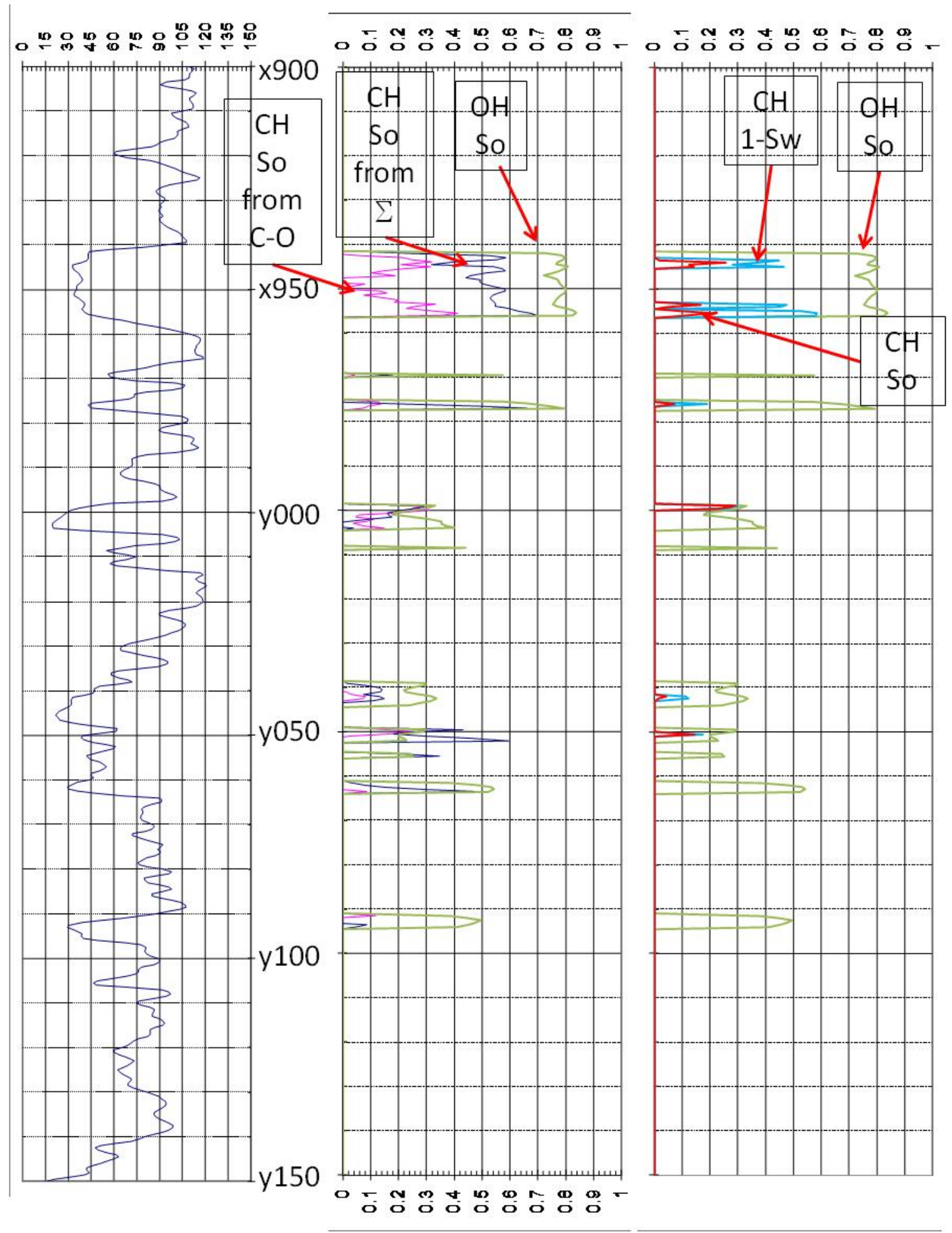

Figure 9: Two-phase saturation values (middle track) and three-phase saturation values (right track) for Well 1. 

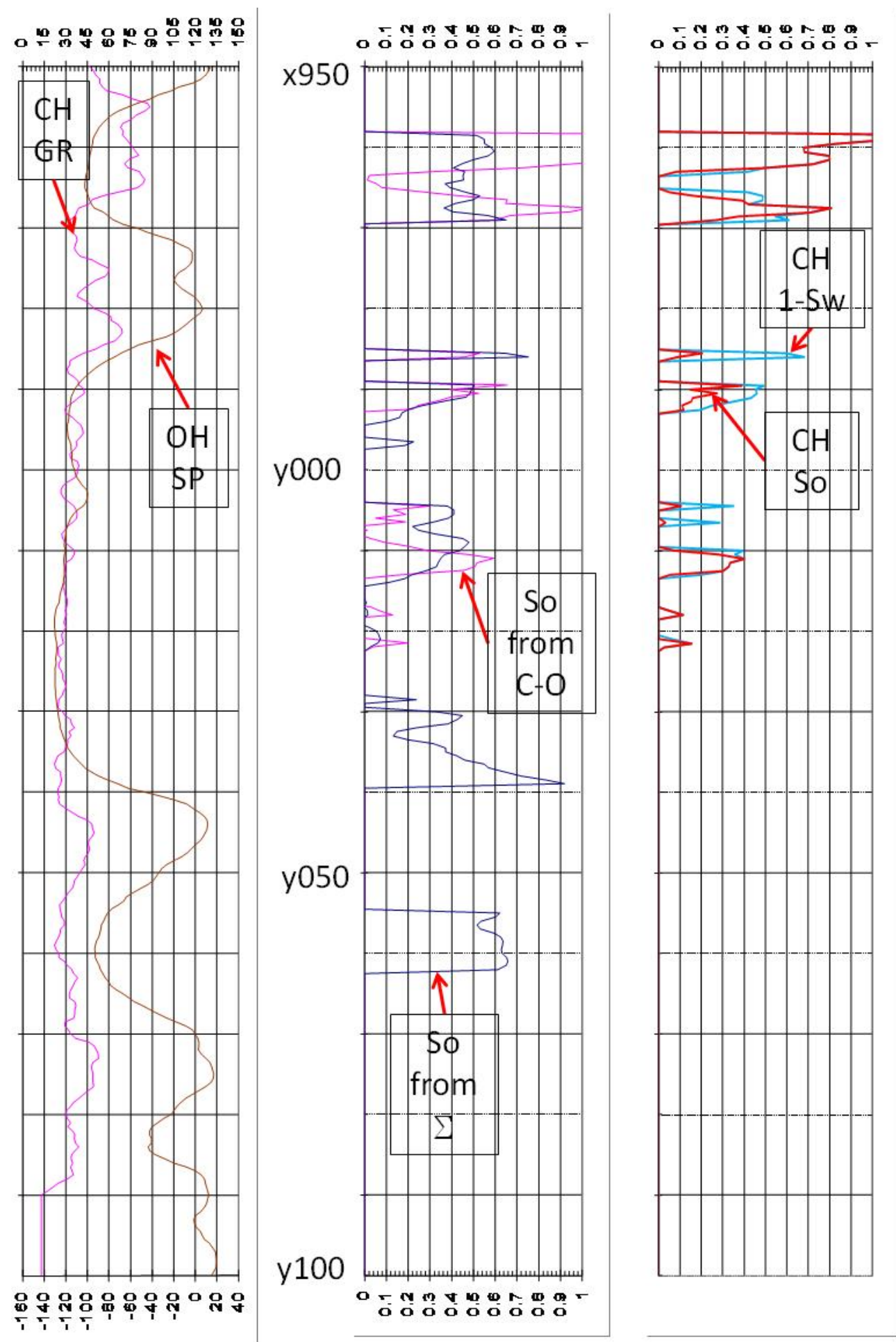

Figure 10: Computed two-phase (middle track) and three-phase (right track) saturations using shale content from the open hole logs for Well 2. 


\section{Subtask 1.3 Simulation Study of a Mature $\mathrm{CO}_{2}$ Flood}

Subtask 1.3 was a simulation study of the Little Creek Field. The simulation study was broken into two pieces: a simulation study of the pilot area to evaluate sweep during a time frame where data quality and quantity was high. This area has been studied previously by both Shell and Arco. In addition, a second area of study in the active portion of the $\mathrm{CO}_{2}$ flood was evaluated. History matches of both the pilot area and the active area were accomplished primarily by mimicking the waterflood that occurred prior to the injection of $\mathrm{CO}_{2}$. Once the waterflood history matches were obtained the $\mathrm{CO}_{2}$ flood was simulated and evaluated based on the actual flood response. After several iterations of modifying the simulation model during the waterflood stage, an appropriate history match of the $\mathrm{CO}_{2}$ flood was obtained. These models were then used evaluate several scenarios for both projecting current operations as well as "what if" scenarios related to evaluating flood patterns and completion techniques.

The Little Creek Field produces $39^{\circ}$ API gravity crude oil from the Q and $\mathrm{Q}_{2}$ sandstones. The average pay zone is at a depth of approximately $10,750 \mathrm{ft}(10,350 \mathrm{ft}$ subsea). The average net thickness of the Lower Tuscaloosa $\mathrm{Q}$ and $\mathrm{Q}_{2}$ sandstones is $40 \mathrm{ft}$. The maximum net thickness of the Q sandstone is $55 \mathrm{ft}$ while it is $30 \mathrm{ft}$ for the $\mathrm{Q}_{2}$ sandstone (Smith, 1973; Werren, et al., 1990). The Lower Tuscaloosa $Q_{-} Q_{2}$ sandstone bodies exhibit "fining-upward" response on electric logs and are interpreted as point bars deposited in a fluvial meander belt on a deltaic plain (Werren, et al., 1990). The age of the reservoir rocks is late Cretaceous, Cenomanian and the lithology is fine to medium-grained sublitharenite (Werren, et al., 1990).

The Q sandstone is the most common reservoir rock and was penetrated by almost all of the wells in the field. The $\mathrm{Q}_{2}$ sand is not present over large sections of the field (Smith, 1973; Werren, et al., 1990). The $\mathrm{Q}$ and $\mathrm{Q}_{2}$ sandstones are distinct markers, but they appear to be contiguous. Based on pressure and production data, it was interpreted that the two sands are in communication (Cronquist, 1968; Werren, et al., 1990). In some wells, the $\mathrm{Q}_{2}$ sandstone, which is the lower layer, disconnects from the $\mathrm{Q}$ sandstone because of a shale zone in between. It merges back with the Q sand in most other parts of the field (Cronquist, 1968). Smith (1973) suggested that there are no data available indicating major discontinuities within the reservoir. In addition, it was also suggested that the continuity of the reservoir was usually obvious based on reservoir performance (Werren, et al., 1990).

The reservoir was initially filled with undersaturated oil and Cronquist (1968) indicated that there was a common water oil contact (WOC) in the field at 10,415 ft subsea. However, Werren, et al (1990) found that "free and 100\%-water levels" were at 10,425 ft and 10,420 ft, respectively based on production data, capillary pressure curves and log data. Cronquist (1968) claimed that there was usually no clean oil below 10,390 ft subsea and defined this depth as the base of the transition zone. A value of 10,415 ft subsea was used as a WOC contact level in our simulation model which will be discussed shortly. Basic reservoir and fluid properties for Little Creek were obtained from Hansen (1977a), Morse (1979) and Youngren and Charlson (1980).

Core data from the Little Creek Field was reviewed. There were 96 cored wells (out of 233 ) in the field (Senocak, et al., 2008). The differentiation between the $\mathrm{Q}$ and the $\mathrm{Q}_{2}$ sand was carried out by reviewing logs from the field. A total of 10 wells were found which had core data from the $\mathrm{Q}_{2}$ sand (Senocak, et al., 2008). The arithmetic mean of the porosity values and the geometric mean of the permeability values including all data points from each cored well are $24.3 \%$, and $33.8 \mathrm{md}$, respectively. For more accurate results, these variables were also calculated using data from 45 wells which had more than 15 measured data points per well. However, the 
results did not change drastically with an arithmetic mean porosity value of $24.1 \%$ and a geometric mean permeability of $32 \mathrm{md}$.

Subsequently, porosity-thickness $(\phi h)$ and permeability-thickness $(k h)$ of the cored interval for each cored well were calculated. Using these core derived $\phi h$ and $k h$ values, maps of these parameters were generated throughout the field using a database and mapping software package called Dynamic Surveillance System (DSS). The variation in porosity affects pattern volumes and original oil in place estimations. Using the pattern average porosities rather than the field average porosity resulted in a 5\% increase in estimated oil in place for the field.

An important factor in the application of a $\mathrm{CO}_{2}$ flood such as Little Creek is an understanding of reservoir heterogeneities and the recognition of the main flow channels in the reservoir in order to evaluate sweep efficiency. Dykstra-Parsons $\left(V_{D P}\right)$ and Lorenz coefficients $\left(L_{C}\right)$ values (Jensen, et al., 2000) were computed for each of the 96 cores in the field. The $V_{D P}$ values range from 0.47 to 0.96 with a mean of 0.45 and a standard deviation of 0.133 . The $L_{C}$ values range from 0.22 to 0.75 with a mean of 0.445 and a standard deviation of 0.11 (Senocak, et al., 2008).

An attempt was made to correlate pattern performance to these heterogeneity measures. For each of the wells in a flood pattern in the field, the heterogeneity measures were compared to oil recovery and gross $\mathrm{CO}_{2}$ utilization (defined as the ratio of the cumulative $\mathrm{CO}_{2}$ injected to the cumulative oil recovered). There was an expected trend that oil recovery generally decreased with increasing values of the heterogeneity measures and tended to be lowest for the highest values; however, there was a large variation which resulted in weak $R^{2}$ values. When gross $\mathrm{CO}_{2}$ utilization was plotted against the Dykstra-Parsons and Lorenz coefficients a slightly better correlation was observed. The Dykstra-Parsons and Lorenz coefficients were not correlated in any sense to EOR recovery or gross utilization when using the last available data rather than a consistent intermediate value for the HCPV injected. The best correlations were found when average porosities for each pattern computed from the core data were used rather than the constant field average porosity of 23.4\%. Standard Single Factor Analysis of Variance (ANOVA) and t-tests to evaluate whether the slopes seen were significant were conducted and suggest that while the $R^{2}$ values are on the low side, they were significant.

Up to this point, it was still hard to see the interpreted channels quite as easily as was hoped. Net sand maps and the interpretation of the depositional setting show a distinct fluvial channel. It was difficult to see any sense of the channel with existing porosity-thickness and/or permeability-thickness maps.

The consistency with the channel models was observed when Dykstra-Parsons and Lorenz coefficients were mapped using a "nearest neighbor" algorithm. Although the interpretation of the channel was subjective, it was consistent with existing geological interpretations (Smith, 1973). The Lorenz coefficient map indicated the channels more clearly than the Dykstra-Parsons map (Senocak, et al., 2008). The interpreted channel system using the Lorenz coefficient map offered a way to determine different rock types in the reservoir simulation model.

The IMEX ${ }^{\circledR}$ software from the Computer Modelling Group (CMG) with the pseudomiscible option is a finite-difference, black oil simulator that was used in this study (Computer Modelling Group, 2007). This is similar to the systems used in previous studies. The pseudomiscible, black oil fluid model is based on the method introduced by Todd and Longstaff (1972) and is used to simulate the miscible displacement performance by representing the reservoir with a coarse numerical grid (Computer Modelling Group, 2007). In order to evaluate the mixing 
capability of the miscible fluids within the grid blocks, a mixing parameter $\omega$ is introduced (Computer Modelling Group, 2007). The $\omega$ parameter ranges from zero to one where a value of one stands for complete mixing of $\mathrm{CO}_{2}$ and oil while a value of zero is for the case of no dispersion (Computer Modelling Group, 2007). For 3D miscible displacement studies, an $\omega$ value of 0.33 is recommended (Todd and Longstaff, 1972) and this value was used for all of the studies in this work. The model consists of four-components which are water as the wetting phase, and gas, solvent and oil as non-wetting "phases". In this simulation study, it is believed that the pseudo-miscible model is sufficient to represent the $\mathrm{CO}_{2}$ displacement process since it is faster and more efficient compared to computationally complex compositional models. The purpose of this work is to evaluate sweep and investigate options to improve sweep. This is viewed as being dominated by displacement. Had this work focused on current operations at Little Creek, the compositional model would be required since the recovery mechanism currently is more likely to be dominated by vaporization of the remaining oil rather than displacement. Complete details of the simulation study are provided in Senocak (2008).

The structure map and the net pay isopach maps of the $\mathrm{Q}$ and $\mathrm{Q}_{2}$ sands were obtained from Denbury Resources, Inc. (Pennell, 2006), in order to incorporate the structure and thickness variation throughout the reservoir into the model. This is somewhat different than what was considered in previous simulation studies at Little Creek where structure and net pay were represented in a conceptual sense, but not explicitly (Morse, 1979; Youngren and Charlson, 1980). The maps were digitized by using the WINDIG ${ }^{\circledR} 2.5$ digitizing software (Lovy, 1996). The digitized maps were brought into the CMG Builder software to begin the process of building the model.

\section{Pilot Area Simulation Study}

In the simulation model for the pilot area, the grid system had considerably more grid blocks than in previous studies. A $14 \times 12 \times 4$ block grid system was used in the Shell studies (Cottrell, 1984; Morse, 1979) while a $10 \times 10 \times 10$ grid system was used in a study by Arco (Youngren and Charlson, 1980). In a later Shell study, Cottrell (1984) added several grid cells near the edges of the pilot area as a sensitivity to the pattern area pore volume. Our three dimensional grid system is $50 \times 50 \times 8$ with the $\mathrm{Q}_{2}$ sand in the last layer of the pilot area of the 20,000 grid cells in our study. A total of 5,607 blocks were outside the reservoir and these grid cells were assigned thickness values of 0 and were effectively removed from the active simulation grid (Computer Modelling Group, 2007). The grid was created as a Cartesian grid system with regular $120 \mathrm{ft} \times$ $120 \mathrm{ft}$ grid cells over a $40 \times 46$ cell area. The remaining grid cells were expanded in an attempt to include extra storativity and flux outside the main reservoir "window". The edge of the field to the east and south was not very well defined, so the most likely reservoir volume for this part of the reservoir was determined after several adjustments to the model. (Senocak, 2008)

A limited water drive mechanism was believed to exist based on the early production performance (Cronquist, 1968). An aquifer was attached to the easternmost side of the model in order to evaluate the nature of the water influx and its influence on the water production. A Fetkovich aquifer model was selected to represent the water influx in the reservoir. Aquifer size and strength were used as history matching parameters because the precise extension and strength were not known. The dimensions and ability to flow were changed until a reasonable history match result was obtained for primary recovery.

Data provided by Denbury Resources, Inc. had some missing production and injection data for the pilot area. Different Shell reports, information from the Mississippi Oil and Gas 
Board and well files were used to compile the production and injection history because no single source had all of the data required from initial production to the end of the pilot. In case of any discrepancies between the reports, data from the Shell reports were used since it was considered to be a more reliable source. Most of the production and injection data were obtained from Hansen (1977a) by digitizing the data from figures showing the daily production and injection for each well because only the cumulative field data was tabulated in his report.

The reservoir crude oil was a highly undersaturated black oil with a stock tank gravity of $39^{\circ} \mathrm{API}$ and an initial gas-oil ratio of $555 \mathrm{SCF} / \mathrm{STB}$. The initial reservoir pressure and the bubble point pressure were 4840 psi and 2150 psi, respectively (Werren, et al., 1990). Initial reservoir temperature was $248^{\circ} \mathrm{F}$ and minimum miscibility pressure provided by Denbury Resources, Inc. was 4500 psi. Note that this miscibility pressure is lower than the 4800 psi used by Youngren and Charlson (1980) and is also on the low side of the $\mathrm{CO}_{2}$-recombined fluid critical point of 4700 psi \pm 300 psi measured by Orr (1976). The miscibility assumption between $\mathrm{CO}_{2}$ and Little Creek crude oil was shown to be valid based on the study of Morse (1979).

In the simulation model, PVT data were obtained from a PVT data analysis by Fair (1987) where he analyzed 72 PVT data points including laboratory analysis of fluid samples collected from different wells between 1958 and 1966. $\mathrm{CO}_{2}$ properties required for the solvent PVT table in the simulation model were obtained from the Chemistry WebBook of National Institute of Standards and Technology (Watters, 2005) at pressure increments of 200 psia from 100 to $5100 \mathrm{psi}$ at $248^{\circ} \mathrm{F}$. The volume and density information obtained from this reference were used to compute the solvent expansion, formation volume and compressibility factors at each pressure.

Much of the initial input data for the reservoir properties were obtained from the previous Shell studies. However, there are some fundamental differences compared to the previous simulation studies in terms of using the data. Instead of using constant values for porosity and permeability throughout the field as was done in the previous simulation studies (Morse, 1979; Youngren and Charlson, 1980), values from the core study at each well were used. Layer by layer porosity and permeability maps were created where the reservoir properties from the core study were first determined for each layer separately. Porosity and permeability values at the well locations were then imported into the CMG Builder ${ }^{\circledR}$ software. Layer porosity and xdirection permeability values for each grid cell in the layer were generated using the Ordinary Kriging estimation method (Computer Modelling Group, 2007). An east-west vs. north-south permeability contrast was taken to be 2 to 1 in the pilot region based on information in Hansen (1977a). Vertical permeability values were assigned by multiplying the x-direction permeability by 0.001 (making $T_{z} / T_{x} \approx 0.58$ ).

Two rock-type zones were defined based on the analysis of well water production values and the map of the Lorenz coefficients in the pilot area indicating that some of the water production could be due to poor quality rock. One region corresponds to a rock-type which was assumed to be of lower rock quality (i.e. more heterogeneous) while the other was an assumed homogeneous region based on the Lorenz coefficient map. The more homogeneous rock region used the same oil and water relative permeability curves as in a study by Fair (1987). In the more heterogeneous rock region, the oil flow was lowered a bit and had much higher curvature. The water relative permeability values were generally lower in this region as well, but with similar curvature as in the work by Fair (1987).

Capillary pressure curves in the high quality rock region were deemed negligible based on work by Cottrell (1984). Oil-water capillary pressures were considered to be essential in the 
more heterogeneous region as evidenced by the interpreted transition zone production in Well 110. This "poor" rock region was located out of the main reservoir channel viewing it as something like an overbank deposit or the edges of the point bars. The capillary pressure curve used by Fair (1987) was adjusted slightly to obtain the correct early water production seen in the field. The capillary pressure relationship in Fair (1987) was obtained by a review of the petrophysical properties of Little Creek by Shannon (1984). In that review, Shannon generated one average curve from the capillary pressure data available from 36 air-mercury measurements on core plugs from Little Creek. It was noted that the relationship was realistic based on saturation profiles viewed in the field (Fair, 1987).

Primary, secondary and $\mathrm{CO}_{2}$ flood responses were simulated and history matched in the pilot area. The reason behind simulating the full history was to provide an estimate of the fluid saturations before the $\mathrm{CO}_{2}$ flood started and to provide an accurate representation of the $\mathrm{CO}_{2}$ response with minimal adjustments of the model to account for the complex response to the $\mathrm{CO}_{2}$ flood. Oil production rates were used as the operating constraints for the history match.

In early versions of the model, 4 layers were used instead of 8 . These initial attempts to match the history in the pilot area under the oil constraint mode resulted in a failure of the model to produce enough water during $\mathrm{CO}_{2}$ flooding. The first acceptable match of the cumulative water production history was obtained when two rock regions based on core analysis were applied into the model. Additionally, gravity segregation of $\mathrm{CO}_{2}$ into the upper layers occurred quite rapidly causing higher simulated solvent rates than was seen in the field data. Based on these results, the model was switched to an 8-layer model and vertical permeability was decreased which provided improvement in both water and solvent production in accordance with historical performance.

The area-wide history match to the cumulative water production between the years of 1958 and 1978 was quite good. In order to verify the model, history matches for each individual well were also done. The actual water production and the simulated values for each well were in good agreement (see Senocak, 2008 for additional plots related to the history matching). Additionally, cumulative gas production for the area and simulated gas production during primary and secondary recovery also matched the actual gas production data reasonably well.

Water production rates for each well were not quite as good as the cumulative rate matches, but they were still quite good and are a better indicator of possible model mismatches compared to the cumulative graphs. For instance, the model started to produce water in several of the wells a little earlier than history. It is also possible that the earliest water production data may not be recorded. The simulated water rates for each well also show that the model missed some peak points of the water production but not by much.

History matching of the $\mathrm{CO}_{2}$ flooding period was more challenging than the waterflooding period. Since it is a "window model" and the pilot area was shut in starting from the end of waterflooding until the initiation of the $\mathrm{CO}_{2}$ flooding, the primary difficulty encountered was accounting for the flux into and out of the model region. This difficulty was overcome by using injection and production wells in several of the outermost grid cells allowing fluids to move across the model area. In the early years (during secondary recovery), these wells were controlled by bottom-hole pressure according to isobar maps from Cronquist (1968). After June, 1964, the BHP constraints for the wells were adjusted based on the water production history and kept constant. Our estimation was that this was the most reasonable way to handle the flux into and out of the model. 
The final model provided a good history match of water production for both the waterflooding and the $\mathrm{CO}_{2}$ flooding periods The overall simulated water production compared to the actual values for individual wells during $\mathrm{CO}_{2}$ flooding were in very good agreement for the individual wells during the $\mathrm{CO}_{2}$ flooding period. Water rates, bottom-hole pressure and water cut values of individual wells during $\mathrm{CO}_{2}$ flooding showed some differences, simulated water rates match the historical water rates slightly better during the $\mathrm{CO}_{2}$ flooding period compared to the waterflooding period.

Although there was no tabulated pressure information in any of the reports provided, there is some information about the reservoir pressure in Cronquist (1968) and Hansen (1977a) mentions that the reservoir pressure in the pilot area was around 4400 psig in 1973 before pilot operations were started. Simulated average reservoir pressure is close to that value in 1973.

The history match results presented relied primarily on two things. First, an adequate primary and secondary recovery match which was controlled by the initial saturation distribution and aquifer support. The two rock regions and corresponding capillary pressure and relative permeability curves provided the solution to the initial reservoir response. Second, since this is a window model, the saturation distribution at the start of $\mathrm{CO}_{2}$ operations is a key. This distribution was controlled by the placement of the injection and production wells outside the main simulation window since this area had good information regarding the pressure and saturation movement from the maps by Cronquist (1968).

\section{Evaluation of Alternative Operations for the Pilot Area}

From the history match it was noted that the well (Well 1-10) located in the lowest part of the reservoir (see Figure 11) had been used for $\mathrm{CO}_{2}$ injection and was in the heterogeneous part of the reservoir based on the channel description from the Lorenz coefficients map discussed previously. The south part of the model still had high oil saturation values and the oil sweep was mainly deployed towards Well 1-11, especially in the top layers. Rapid response to the $\mathrm{CO}_{2}$ flood and consequent oil breakthrough in Well 1-11, as well as previous reservoir simulation studies, provided evidence that the east-west permeability values were larger than the northsouth values. Solvent saturation profiles showed that in the top layers, the $\mathrm{CO}_{2}$ continues beyond Well 1-11. The reason for this could be that the east-west vs. north-south permeability ratio is 2 to 1 but it could also be that Well 1-11 was not completed in these layers.

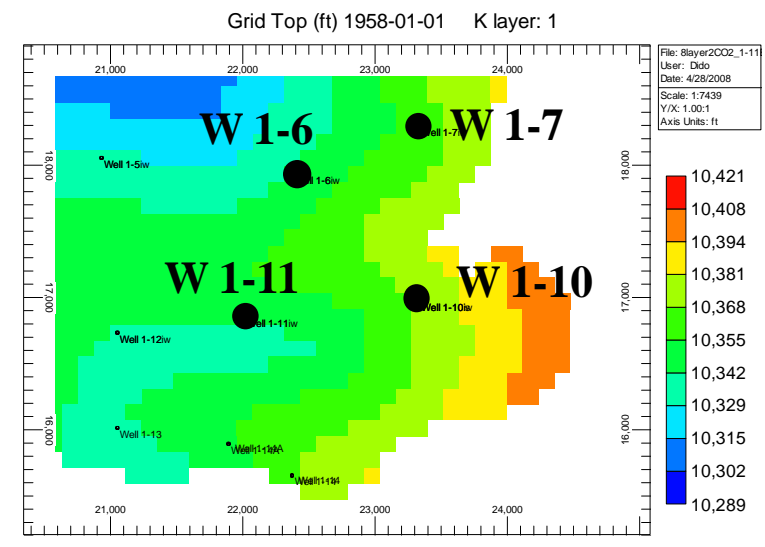

Figure 11: Structure Map of the Top of the Formation in the Pilot Area 
Based on these observations, alternative cases were constructed to compare the sweep efficiency under 34 different scenarios. In all cases, the original pattern geometry of $1 / 4$ of an inverted nine-spot was used and the five water injection wells that contained the flood (Well 1-2, $1-3,1-4,1-5$ and 1-12) were kept as they were in the history match. All cases used the historical $\mathrm{CO}_{2}$ injection rates as the constraints on the injectors with a BHP constraint of 5000 psi on the producers (to keep the flow above the MMP).

The first 9 scenarios tested the continuous injection process with different wells in the pilot as the injector. Each of the three wells was evaluated in three ways. First, no modifications to the existing well configurations were made other than a different well was used as the injector in the pattern. Second, several of the wells had perforated intervals that did not penetrate all of the layers in the model. Therefore, these layers were opened to flow and the model re-run to obtain recovery from the area. Third, there were three wells in the southern part of the reservoir that were salt water disposal wells but located in a manner where there was available oil to be swept in the pattern zones. These wells were "converted" to water injection to sweep this trapped oil towards the producers and the model was re-run.

For the continuous injection cases tested, only injection into Well 1-6 recovered more oil than was seen in the original pilot using the existing completion configurations. The original pilot area recovered a cumulative 2.2 MMstb or about $110,000 \mathrm{stb}$ of incremental oil while the 16 injection recovered an additional 20,000 stb over the pilot recovery. Changing the completion intervals and adding the southern injectors caused all of the continuous injection scenarios tested to recover between 80,000 and $100,000 \mathrm{stb}$ of oil over what the original pilot recovered. Using Well 1-11 as the injector had the highest oil recovery of the 9. In general, the significantly higher oil recoveries seen in these scenarios were due to moving oil out of the southern area and by completing additional layers to allow more solvent to enter and sweep oil out of those layers. These both may be model artifacts; however, modeling to observe the remaining oil saturations provided the capability to modify reservoir development plans and observe oil possibly trapped in parts in the reservoir.

Since Well 1-10 was situated in a structurally low position and aligned with a permeability streak, changing injectors may result in gravity helping to spread the solvent more evenly when a structurally higher injector is used. In addition, because of the permeability and porosity distributions in the reservoir, the swept area is simply different than in the original pilot. Rather than sweeping oil away from the lower boundary, these other wells are using the bounding water injection and the water influx to focus and constrain the $\mathrm{CO}_{2}$ into the pattern area. For all of the cases, more of the solvent seems to be staying together (the solvent saturation values in each layer appear less diffuse than in the pilot model) and the solvent seems to be staying within the region of study (very little $\mathrm{CO}_{2}$ is lost "outside" of the pattern area) more than in the pilot model especially in the upper layers.

Finally, several of the tests likely could have continued to recover more oil as either breakthrough had not occurred in the producer wells or GOR values had not yet reached the critical threshold where Shell decided to shut down the pilot. In these studies, injection ceased when the total amount of $\mathrm{CO}_{2}$ injected into the pilot injection well ceased to ensure a reasonable comparison.

Undesirable mobility ratios due to the low viscosity of the displacing fluid compared to the displaced fluid cause poor sweep efficiency (Green and Willhite, 1998). In 1958, Caudle and Dyes developed the water-alternating-gas (WAG) process by suggesting the injection of water and gas alternately to alleviate this problem (Green and Willhite, 1998). WAG injection ratios 
generally range from 0.5 to 4 volumes of water per volume of solvent at reservoir conditions (Green and Willhite, 1998).

In Case 4, an attempt was made to understand how the oil sweep might be affected if the pilot area was operated using a WAG technique. Five different simulation runs looking at the impact that WAG ratio and WAG cycles have on recovery for each of the possible injector wells were performed. Both $\mathrm{CO}_{2}$ and water were injected in the same well in cycles. In the first three runs, a WAG process was simulated consisting of one year $\mathrm{CO}_{2}$ injection followed by one year of water injection performing at WAG ratios of 1:1, 1:2 and 4:1 respectively. The amount of $\mathrm{CO}_{2}$ injection was always kept the same as in the original pilot, and the injected water amounts were calculated based on the specified WAG ratio. In the last two runs, simulations of 3 months of $\mathrm{CO}_{2}$ injection and 3 months of water injection were followed by one month of $\mathrm{CO}_{2}$ injection and one month of water injection at a WAG ratio of 1:2.

The results of these scenarios for each different $\mathrm{CO}_{2}$ injector indicate that one year of $\mathrm{CO}_{2}$ injection and one year of water injection produce much more oil than was seen in the original pilot. Different WAG ratios with this cycle did not change the oil recovery significantly, but did increase the water cut. More frequent cycles of WAG injection with a 1:2 WAG ratio increased the oil recovery more for each of the different injectors. Figure 12 shows a comparison of the different $\mathrm{CO}_{2}$ injectors in terms of cumulative oil production as a function of time. The best oil recovery is obtained when Well 1-7 is used as a $\mathrm{CO}_{2} /$ water injector with a 1:2 WAG ratio in one month cycles.

Solvent saturation maps show that the $\mathrm{CO}_{2}$ in the WAG cases tends to move away from the injectors in a nearly radial pattern in all layers with some layers having solvent move a bit faster due to higher permeabilities. This contrasts with solvent saturation maps for several of the continuous injection cases which showed a more oval appearance rather than radial. This seems to show that the WAG process stabilizes the solvent front providing a more favorable mobility contrast to increase the sweep efficiency.

The WAG process was also simulated using Well 1-10 as the WAG injector without any additional operations. The aim was to understand the difference between continuous $\mathrm{CO}_{2}$ injection and the WAG process under original operating conditions. The incremental recovery for this test was approximately $177,000 \mathrm{bbl}$ during $\mathrm{CO}_{2}$ flooding. Oil is recovered at water cut values relatively consistent with history. Solvent production was earlier than historical data, but was much lower than history from late 1975 to late 1976. Thus the pilot area under these modeled circumstances would recover nearly 70,000 bbl more oil using the WAG process rather than continuous $\mathrm{CO}_{2}$ flooding.

Rao, et al. (2004) mentioned that the field performance of WAG floods have been disappointing and have yielded only 5-10\% increases in oil recoveries. As an alternative method to the WAG process, the Gas-Assisted Gravity Drainage (GAGD) process was introduced by Rao, et al. (2004). An attempt to simulate the application of the GAGD process in the pilot area was developed as Case 5 and 6 additional runs were made using each of the pilot wells as a horizontal or vertical injector in the uppermost layers.

The GAGD process attempts to take advantage of gravity effects by providing vertical segregation between the injected $\mathrm{CO}_{2}$ and the reservoir oil. This process uses horizontal production wells near the water-oil contact and existing vertical $\mathrm{CO}_{2}$ injection wells (Rao, et al., 2004). The purpose of converting the producing wells to horizontals was to see if this would enable $\mathrm{CO}_{2}$ to contact larger amounts of unswept oil in the reservoir, taking advantage 


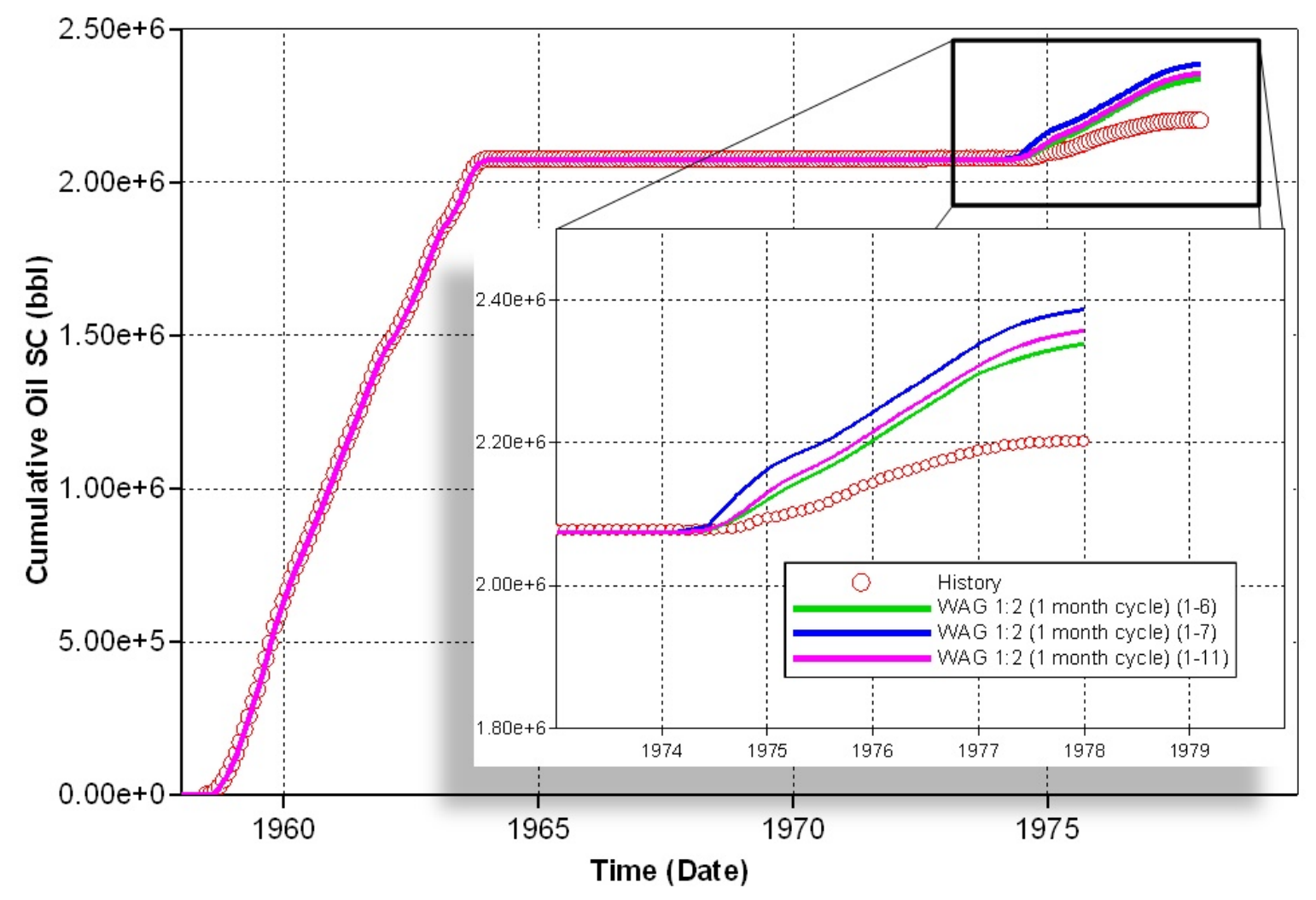

Figure 12: Comparison between the best recoveries from all runs for each injector

of the gravity override phenomena (Kuuskraa, 2008). According to theory, the volumetric sweep efficiency would be maximized by a $\mathrm{CO}_{2}$ zone moving down and to the sides, providing more sweep of the reservoir. Rao, et al. (2004) also state that the natural gravity segregation would assist delaying, or eliminating breakthrough to the production well.

For these runs, the models were built with all of the layers available as necessary and with the southern water injectors active. In the first set of runs, the vertical production wells were converted to horizontal wells completed in the center of the $6^{\text {th }}$ layer in order to stay away from the water-oil contact. The $\mathrm{CO}_{2}$ injection well was a vertical well but was completed only in layers 1 to 4 . The second set of runs used horizontal injection wells completed in the top zone to further take advantage of the $\mathrm{CO}_{2}$ segregation process.

The well constraints were the same as previously defined in order to be consistent. The constraints for the $\mathrm{CO}_{2}$ injection well was the historical injection rate and the constraints for production wells were bottom-hole pressures of $5,000 \mathrm{psi}$. Several test models were simulated to predict the reservoir reaction to changes in the lengths and orientation of the horizontal wells. Considering the distances between the wells (especially between Wells 1-7 and 1-10 when they were used as production wells), a 1,200 ft length of horizontal section was used for horizontal production wells in each model. Laterally completed horizontal wells along the structure yielded 
the best recoveries. However, for the last three runs $360 \mathrm{ft}$ length horizontal sections provided more recovery.

The responses for each run for three different horizontal and vertical injectors are shown in Figure 13. In the best scenario, Well 1-7 was a horizontal injector in the top layer, and 213,350 barrels of oil during $\mathrm{CO}_{2}$ flooding could be recovered. For vertical injectors, the highest incremental oil production is about 193,100 bbl when Well 1-6 was used as the injector and Wells 1-7, 1-10 and 1-11 were used as horizontal production wells. Note that the water cut levels are relatively lower than history in this scenario. This confirms the idea that sweep will be enhanced in the reservoir without an increase in water production as suggested by Rao, et al. (2004). Also note that solvent rates are generally lower than historical values until early 1977 (except when Well 1-7 was used as both a vertical and a horizontal injector). However, the production forecasts for Case 5 were not as beneficial as was hoped. Although the GAGD process was developed as an effective alternative to WAG, the simulated WAG cases produced more oil (almost 310,000 barrels of oil when Well 1-7 was a WAG injector vs. the 213,350 for the GAGD case). As with the WAG case, there is likely even more oil that could be recovered and the ultimate plateau for the cumulative production could be a bit higher since high solvent production rates and/or high GOR values have not been encountered yet.
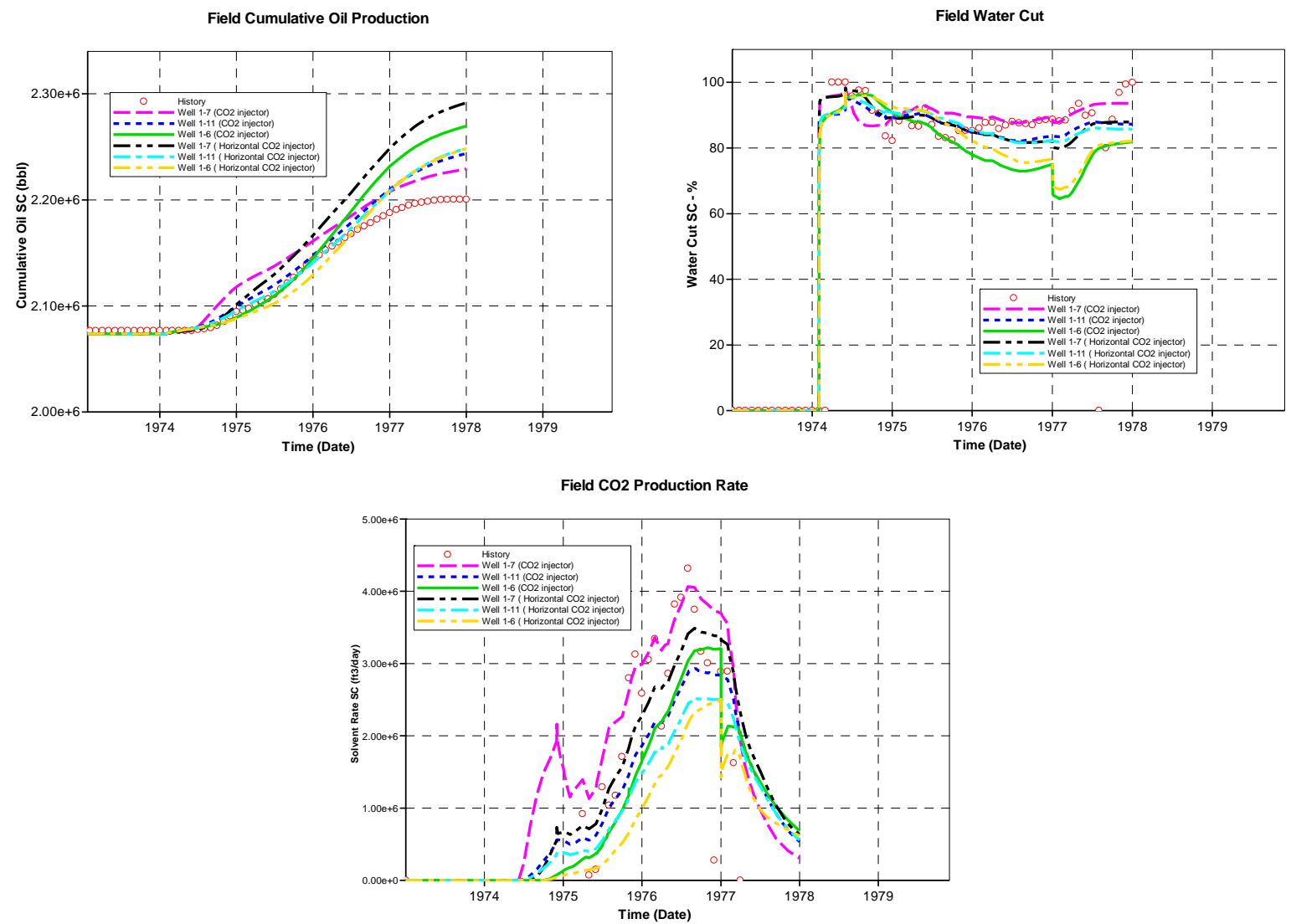

Figure 13: Field Cum Oil, Water Cut and $\mathrm{CO}_{2}$ Rate for GAGD Evaluation 
Rao, et al. (2004) mention that gravity-stable gas injection would be best if applied in low connate water saturation, thick, highly dipping or reef type light oil reservoirs with moderate to high vertical permeability. However, Little Creek has a high connate water saturation (0.56) and might not dip enough to be able to take advantage of the natural gravity segregation. Note that the vertical permeability values used in the model were fairly low in order to provide the history match. This might be another explanation for the lower oil recovery results. The gravity number for the pilot area was around $10^{-6}$ when average values for reservoir and fluid parameters were used. Thus, the lower recovery results are not a surprise. It is encouraging that if an optimal well configuration can be determined, then the GAGD and WAG results might be similar for fields like Little Creek that have low structural relief and that may be sensitive to water injection.

A cross-sectional view of the solvent saturation for each layer between wells for the WAG configuration showed that the solvent generally migrates through the middle layers during the flood with only small pockets of high solvent saturation in layers with higher permeability. This showed that the solvent does not channel to the producer and the WAG process seems to be working as in theoretical pictures of the process. A similar cross-sectional view of the GAGD configuration also appeared to be working as expected. The solvent was staying primarily in the top layers and gradually migrated down to the producer (Senocak, 2008).

\section{Currently Active Area Simulation Study}

After the successful work done in the previous simulation study, it was believed that the understanding gained from modeling the pilot area could be used as leverage for other parts of the reservoir. Pattern 10-9 was chosen because it is one of the active regions in the field and because there was injection survey information which could be used to compare simulation to actual results other than productivity.

Similar to what was used in the pilot area study, the model for this pattern consists of a three dimensional $50 \times 50 \times 8$ Cartesian grid system (base grid cell sizes were $120 \mathrm{ft} \times 120 \mathrm{ft}$ ) The $\mathrm{Q}_{2}$ sand was again located in the bottom layer. Of the 20,000 cells in the model, 17,046 were active. A $45 \times 45$ areal section of the reservoir contains the main portion of the pattern and the remaining grid cells were enlarged using the same reasoning as in the pilot area model. The location of the aquifer attached to the southernmost side of the model was adjusted based on the stated water-oil contact due to a lack of information about the aquifer. The same aquifer properties giving the best history match in the pilot area. The production and injection data provided by Denbury were used for Pattern 10-9 because there were no other published studies related to this area. Fluid and $\mathrm{CO}_{2}$ properties were the same as in the pilot area. Figure 14 shows the grid system used for Pattern 10-9.

Development of the model for Pattern 10-9 was much easier than the pilot area because the experience of modeling based on the core study was already acquired. Porosity and permeability values available at each well were calculated layer by layer in order to create their maps as was done previously. The Ordinary Kriging estimation method was again used for this region. The idea of the two rock types set according to the Lorenz coefficient map was applied, and the same relative permeability and capillary pressure curves for the two rock types were used in this model. Nearly the entire Pattern 10-9 lies inside the channel. 
Grid Top (ft) 1958-01-01 K layer: 1

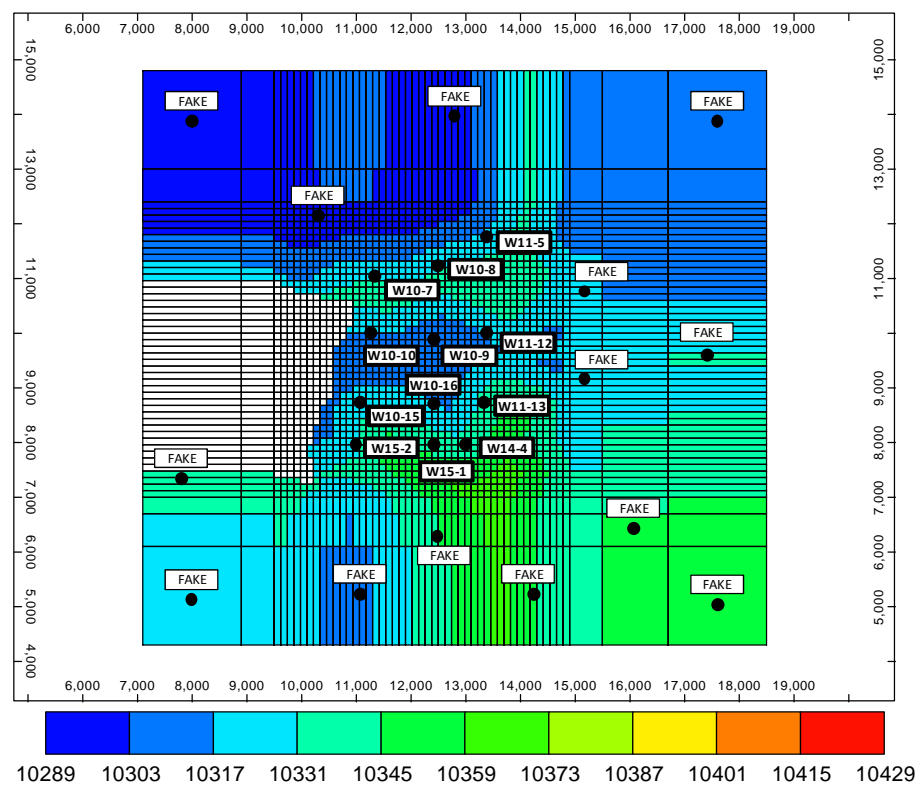

Figure 14: Cartesian grid system used in the simulations for Pattern 10-9

The history matching in this pattern may lead to specific recommendations on active field operations that can improve the sweep efficiency. So, it is important to get a model reasonably matching the historical data to evaluate new strategies. As was done in the pilot area, oil production rates for the production wells, and $\mathrm{CO}_{2}$ for the injectors were used as the operating constraints and the historical water production data was used as the match data. Due to the lack of pressure data in this area, the simulated results will be used to check whether producing bottom-hole pressures were above the minimum miscibility pressure.

Pattern 10-9 is an inverted nine spot pattern that becomes more of a line-drive orientation over time. Well 10-9 was the initial $\mathrm{CO}_{2}$ injector for this pattern. Wells 11-5 and 11-12 were converted to injector wells when they "gassed out". There was no water injection in this particular region, but it is still obvious that waterflooding in areas surrounding Pattern 10-9 had a significant effect. Water injection well locations and flood fronts were based the maps from Cronquist (1968) which showed a line where there was a 10\% water-cut along with estimated reservoir pressure contours. These were clear indicators that the fluid front was moving towards the Pattern 10-9 area. The sweep of oil from the north half and from the south part of the field allowed oil to enter the Pattern 10-9 area during the waterflooding period. In addition, $\mathrm{CO}_{2}$ flooding operations in adjacent patterns were being developed at the same time as Pattern 10-9. Unlike the pilot area, it was very hard to supply the fluid flow from adjacent regions in as systematic a manner. This issue was resolved by setting water and oil injectors and producers at select locations in the expanded portions of the grid system to provide extra fluid inflow and outflow. These wells are shown in Figure 14 labeled as "Fake" and were controlled by bottomhole pressure according to the isobar maps provided by Cronquist (1968) as was done in the pilot area study. After June, 1964, the bottom hole pressure constraint on these wells was adjusted according to water production history and noted operational changes in adjacent wells. Note that the "extra" wells did not have continuous injection or production. They were one or the other at 
different times depending on the operational history, and the isobar maps from Cronquist (1968). Again, the saturation distribution prior to $\mathrm{CO}_{2}$ operations in the area is a key to obtaining reasonable match results.

The area-wide history match to the cumulative water production from the productive history of Pattern 10-9 was matched quite successfully. History matching results were generally similar in quality to those in the pilot area (see Senocak, 2008 for complete details). The history matching for Pattern 10-9 provides the basis from which to test the model under different operating scenarios and investigate how the reservoir response in Pattern 10-9 would be affected by these changes.

The progress of evaluation of the alternatives in this pattern area followed the same reservoir analysis methods as was done in the pilot area alternative operations section. First, the areal oil saturation profile predicted at the end of the history match simulation was examined to see the swept and unswept portions of the pattern. The simulator was run using all available production and injection data through May 2006 (the time of initiation of this study). Additionally, the $\mathrm{CO}_{2}$ saturation distribution at the end of the history match was also observed. The $\mathrm{CO}_{2}$ reached a large portion of the formation in all but the top three layers. However, the amount of solvent deployment decreases gradually in the top layers, possibly due to the relatively lower permeability values in these layers. Geological properties of the reservoir seem to help control the gravity effects in this part of the field more than was seen in the pilot area.

$\mathrm{CO}_{2}$ injection profile logs were run in the $\mathrm{CO}_{2}$ injection well (Well 10-9) over the perforated interval in November, 2006 (see Figure 6 and Figure 7). Injection profile logs determined that the upper $35 \mathrm{ft}$ and the lower $5 \mathrm{ft}$ were taking $28 \%$ of the injected $\mathrm{CO}_{2}$, and that a $10 \mathrm{ft}$ section (20\% of the perforated interval) in between was taking the remaining $72 \%$ of the solvent. This indicates that the injected $\mathrm{CO}_{2}$ may be by-passing much of the oil saturated part of the upper zones. Solvent flux magnitude at reservoir conditions is the output parameter from the simulator that provides values similar to compare to the log response. This parameter is plotted in Figure 15 and shows that the majority of the solvent goes into layers 4-7 (the lower 57\% of the reservoir). Based on the last data values in this figure, $73 \%$ of the solvent flux flows into the lowest $57 \%$ of the reservoir. This is somewhat different from the log result since there is no high injectivity zone above a low injectivity zone at the bottom of the formation. It is likely that there is a higher permeability zone that does not appear in the model since there is no core data for Well 10-9. However, the results are consistent in that the lower parts of the reservoir appear to be taking most of the $\mathrm{CO}_{2}$. Consequently, there is probably still remaining oil especially in the upper layers. Note that the $\mathrm{CO}_{2}$ injection wells are located in the structurally higher parts of the pattern and that all the wells are inside the interpreted main channel. Because of this, the original injection operations may be providing good sweep. However, the original well configuration for the pattern, or the miscible displacement process used might cause poor sweep efficiency.

Based on these observations and the knowledge gained during alternative operations part of the pilot area study, different cases with different scenarios were developed in order to evaluate options for improving the sweep efficiency. Two different miscible $\mathrm{CO}_{2}$-water displacement processes using three different pattern geometries were used to evaluate the increase in oil recovery from Pattern 10-9: (1) WAG flood using the original nine-spot pattern, (2) continuous $\mathrm{CO}_{2}$ injection and WAG using a five-spot well pattern, and (3) continuous $\mathrm{CO}_{2}$ injection and WAG using a line drive pattern.

Three groups of well configurations were used: (a) vertical injection (V.I.) and vertical production (V.P.) wells, (b) vertical injection (V.I.) and horizontal production (H.P.) wells, and 
Well 10-9 Flux Solvent Magnitude

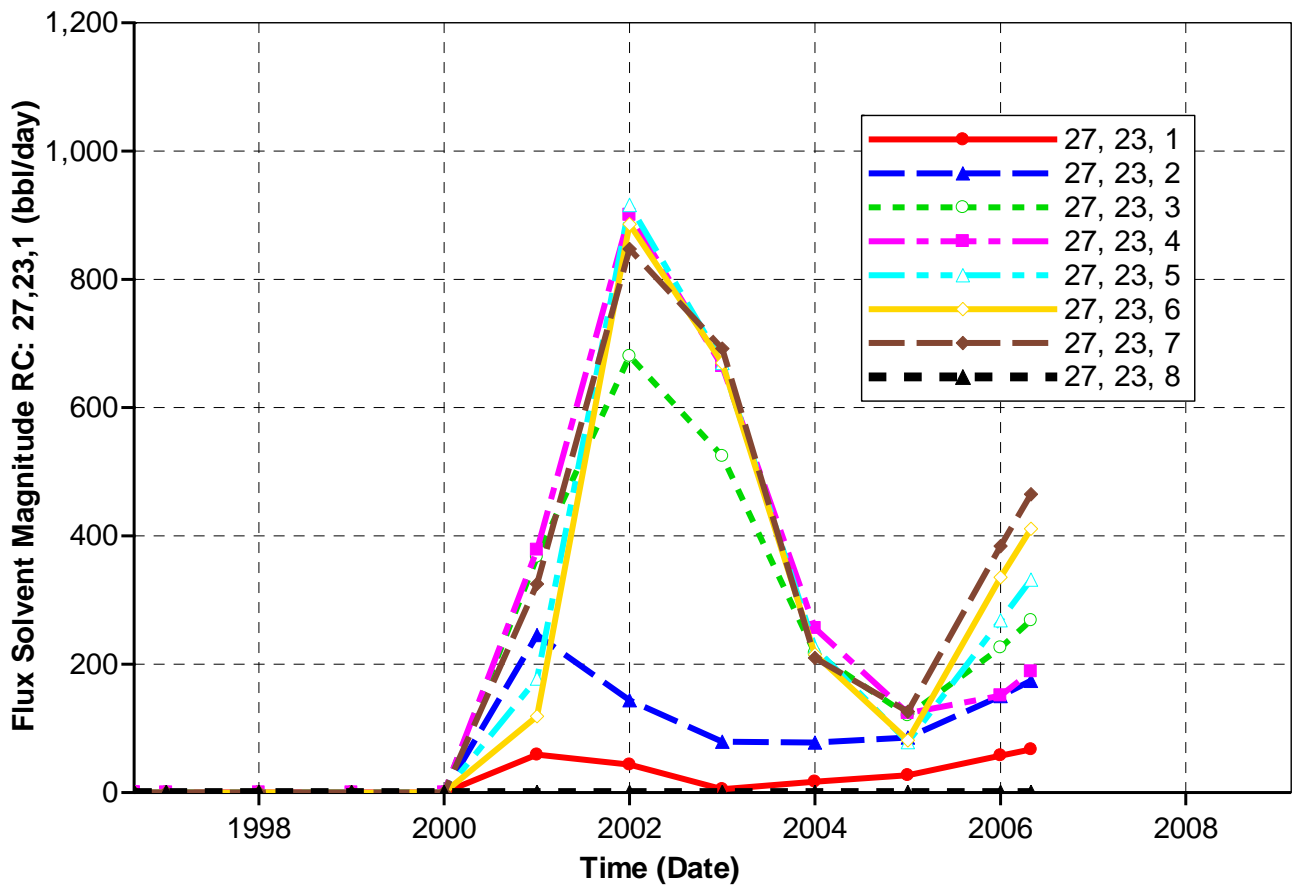

Figure 15: Flux Solvent Magnitude for Well 10-9

(c) horizontal injection (H.I.) and horizontal production (H.P) wells. Horizontal production wells were completed in the sixth layer, whereas horizontal injection wells were perforated in the first layer as was done in the pilot area study. Unlike in the pilot area study, during $\mathrm{CO}_{2}$ flooding, the bottom-hole pressures that provided the best history match for each production well were used as the operating constraints in all cases for this study. In this way, oil recovery comparisons under current operations versus the different scenarios would be consistent. In addition, the GAGD process showed some possibilities for improving sweep and recovery in the pilot area so an evaluation of this process in this pattern area was also done. Finally, an evaluation of other patterns (five-spot and line drive) was also conducted.

For the Pattern 10-9 area, the WAG technique using V.I. and V. P. well configurations provided the highest recoveries similar to the pilot area. The highest oil recovery was obtained when using the existing pattern geometry $(6.7 \%$ OOIP). When the pattern was changed to a $5-$ spot pattern, the ultimate recovery decreased to $6.5 \%$ OOIP, but still was higher than historical values ( $6 \%$ OOIP). As mentioned in the pilot study discussion, the GAGD process has been developed as an alternative method to the WAG process. The process of using horizontal production wells near the bottom of the reservoir and horizontal injectors at the top (instead of vertical ones) can provide good recovery, but success depends on the pattern chosen. The results were positive using the existing pattern geometry and the five-spot but were negative for the direct line drive pattern. 


\section{Summary}

This work has dealt with the evaluation of sweep efficiency in Little Creek Field in order to understand the mechanisms controlling sweep in a late-in-life, continuous injection $\mathrm{CO}_{2}$ flood and search for alternatives to the way the field was conducted in order to improve recovery. In the first part of this work, core analysis and evaluation of heterogeneity effects on reservoir performance were discussed. Dykstra-Parson and Lorenz coefficients were computed pattern by pattern to find a reasonable correlation between oil recovery and $\mathrm{CO}_{2}$ utilization. There was an expected trend showing that the more the heterogeneity, the higher the amount of $\mathrm{CO}_{2}$ utilization and the less the amount of oil recovery. The results did not show perfect correlations, but the relationship between heterogeneity measures and reservoir performance values were shown to be statistically significant by using the ANOVA method and the standard t-tests on the significance of the slope of the regression line.

Contrary to the use of field-wide averages, mapping of well by well heterogeneity measures was shown to be a good tool to see geologic trends when compared to traditional maps. The characterization of the main body of the channel in Little Creek Field was performed qualitatively by using heterogeneity measures. The Lorenz coefficient map provided more insight into the reservoir than trying to map permeability, porosity or thickness alone.

The geological trends observed in the Lorenz coefficient maps were then successfully used to adjust rock-types and guide geostatistical modeling of permeability and porosity when performing reservoir modeling and history matching in the second part of the work. Two rock regions were defined based on the water production values and the map of the Lorenz coefficients in the pilot area. One of these regions was perceived to be heterogeneous and located outside the main reservoir channel, whereas the other was described as being homogeneous or more specifically of a higher quality. Gravity effects were evaluated by using eight layers in the model. After obtaining successful history matching results, the same method developed in the pilot area was used to assess flood response for the Pattern 10-9 area and again good matches for this particular part of the field were obtained.

One of the main keys to both matches was determining the saturation distribution in the reservoir prior to $\mathrm{CO}_{2}$ injection. In these window models, this was accomplished by adding injection and production wells located in the larger volume grid blocks along the outer edges of the models. These wells were controlled based on observed operational changes in the field surrounding the window unless there was something within the window area that was specified.

From the pilot area history match, it appears that a fairly large amount of $\mathrm{CO}_{2}$ moved out of the flood area and was not utilized effectively. Using one of the other pilot area wells that are structurally higher generally allowed more $\mathrm{CO}_{2}$ to stay in the area of interest. Application of the WAG technique increased recovery in the pilot area with reduced utilization rates. A WAG ratio of 1:2 with one month WAG cycles was found to provide the highest recovery values of those tested. From the solvent saturation maps, flood front stabilization appears to be the reason for the higher recoveries seen in the WAG simulations. Simulations of the GAGD process were highly dependent on well orientation and length. When well orientation and length are correctly determined, the simulations of the GAGD process showed slightly lower recoveries than the WAG process simulations, but were fairly close. Given that Little Creek has low structural relief and high connate water saturation, the fact that GAGD technique may have some application in this type of environment was a bit unexpected.

The Pattern 10-9 area simulations showed many of the same characteristics as the Pilot Area simulations. In addition, a five-spot pattern configuration was evaluated. The five-spot 
showed slightly higher recoveries under continuous injection constraints but with lower initial response rates. Recoveries using the WAG technique at the 1:2 ratio were slightly lower than the current inverted nine-spot simulations.

Recommendations for future work include incorporating any additional injection or production profile logs that may have been run in the field into the geological model. As shown in the Pattern 10-9 area model, this data can have a significant impact on the interpretation of the results. Logs have been run in several other parts of the field. Thus use of the techniques from this work should apply to other parts of the field, and should be done. In addition, with current computational capabilities, it may be possible to do a full-field simulation of the Little Creek. Again, techniques from this work should provide a good starting point for that work and reduce the time spent integrating data. 


\section{Task 2: Extension of Sweep Efficiency Findings to Target Reservoirs for $\mathrm{CO}_{2}$ Flooding}

Task 2 focused on extending the findings of Task 1 to reservoirs that may be $\mathrm{CO}_{2}$ flooding candidates. This includes reservoirs with light oils comparable to Little Creek Field and heavy oils (10-20 $\left.{ }^{\circ} \mathrm{API}\right)$ where the potential for $\mathrm{CO}_{2}$ flooding needs additional study, especially relative to sweep effects due to the viscosity variation between the $\mathrm{CO}_{2}$ and viscous reservoir oils. DRI has several target reservoirs in Eastern Mississippi that will serve as candidate reservoirs for this task.

There were two subtasks proposed. Subtask 2.1 was to apply the lessons learned from the Task 1 efforts to light oil systems primarily based on simulation studies which have been completed. Subtask 2.2 was a feasibility study of $\mathrm{CO}_{2}$ flooding in heavy oil reservoirs. The proposed efforts were to be laboratory (primarily PVT) studies as well as simulation studies of the heavy oil $\mathrm{CO}_{2}$ flood process. Again, with the transfer of the project to LSU, the focus changed to primarily a simulation study. However during the transfer Denbury Resources had initiated a heavy oil flood and had problems with almost immediate breakthrough. Therefore the simulation work went from being primarily a theoretical evaluation of heavy oil flooding to a fairly practical one. This work has not been completed yet, but is ongoing.

\section{Subtask 2.1 Light Oil Displacement by $\mathrm{CO}_{2}$ Flooding}

Reservoir simulation was used to evaluate the effect that pattern type and reservoir and fluid parameters have on the effectiveness of a flood. In this work, a number of generic simulator models were used to evaluate the performance of the miscible carbon dioxide displacement process in oil reservoirs. The basic data for simulation models used in this study were taken from geological, rock, and fluid data of the Little Creek Field, Mississippi as were injection constraints that the simulator was trying to mimic.

The particular objectives of the research are: 1) to examine the sweep efficiency of miscible $\mathrm{CO}_{2}$ floods with different injection schemes, including five-spot, nine-spot, inverted nine-spot, and modified inverted nine-spot; 2) to observe the effect of injection rate for a miscible $\mathrm{CO}_{2}$ flooding project; 3 ) to evaluate completion methods that can improve the sweep efficiency; 4) to investigate the influence of reservoir parameters such as oil viscosity, vertical permeability, formation thickness, and well spacing on oil recovery. All of these parameters were seen as important in the Task 1 work except the oil viscosity which is known to be important for the evaluation of heavy oil systems.

The reservoir model was created using the basic properties of the Little Creek Field. In the base case model, miscible carbon dioxide flooding was performed on a square pilot area of 160 acres (1320 ft by $1320 \mathrm{ft}$ ). The horizontal grid system is 33 by 33 cells. A uniform reservoir thickness of $30 \mathrm{ft}$ was assumed with no elevation variation used to control the effects of gravity.

Water, oil, and carbon dioxide were present in the reservoir. Under reservoir conditions of $250^{\circ} \mathrm{F}$ and 5,000 psi, there was a difference in density of the reservoir fluids. Therefore, overriding/under-running phenomenon was expected to occur. To capture the effects of density differences, an analysis of the vertical gridding was performed. From this analysis, it was 
determined that a 6-layer model satisfied the requirements of this study and provided suitable accuracy and limited computational time to a reasonable level.

This study examined several common production/injection schemes for miscible carbon dioxide floods. They are five-spot, nine-spot, inverted nine-spot, and modified inverted nine-spot patterns. The modified inverted nine-spot pattern initiates injection of $\mathrm{CO}_{2}$ exactly as the normal inverted nine-spot model. The only modification is the conversion of production wells to $\mathrm{CO}_{2}$ injection wells when they reach a gas oil ratio (GOR) limit of 50,000 scf/bbl (similar to what was seen in the Task 1 work).

Injection schemes, including the five-spot, nine-spot, inverted nine-spot, and modified inverted nine-spot, were applied to the same model area with the properties of the base case. In these cases, all wells were completed throughout the reservoir section. The modified inverted nine-spot was found to be the most efficient recovery method as it yielded the highest oil recovery.

To determine if similar conclusions would be drawn for other reservoir or operating conditions, a series of simulations were undertaken for different properties of vertical permeability, oil viscosity, and injection rates. In all cases examined, the modified inverted ninespot pattern had the highest oil recovery regardless of reservoir properties and operating conditions. In addition, the 5-spot and modified inverted 9-spot had similar carbon dioxide utilization rates. From the cases studied and under the conditions of the reservoir model, it appears that the modified inverted nine-spot pattern is the most effective strategy for the miscible carbon dioxide flooding process under the constraints of this study.

\section{Completion Techniques}

Since gravity overriding of $\mathrm{CO}_{2}$ may occur in miscible carbon dioxide displacement processes, the perforation method is considered to minimize detrimental effects of this behavior. Under reservoir conditions in this study, the specific gravity of water, oil, and carbon dioxide are $1.02,0.83$, and 0.66 , respectively. Thus, $\mathrm{CO}_{2}$ has a tendency to move upward in the reservoir due to gravity effects. The studied completion techniques and their oil recovery results are shown in Table 2. From the resulting recovery and $\mathrm{CO}_{2}$ utilization results it appears that there is an optimum completion strategy for miscible carbon dioxide displacement. In the case of the homogeneous system analyzed, that strategy is to complete both the injector and producer wells in the lower portion of the reservoir.

Table 2: Completion Strategy for $\mathrm{CO}_{2}$ injection/production.

\begin{tabular}{|c|l|l|}
\hline Completion Strategy & Injectors & Producers \\
\hline 1 & Entire interval, 6 layers & Entire interval, 6 layers \\
\hline 2 & Top 2 layers & Top 2 layers \\
\hline 3 & Middle 2 layers & Middle 2 layers \\
\hline 4 & Bottom 2 layers & Bottom 2 layers \\
\hline 5 & Bottom 2 layers & Top 2 layers \\
\hline 6 & Top 2 layers & Bottom 2 layers \\
\hline
\end{tabular}




\section{Vertical Permeability}

Since gravity segregation has a significant effect on miscible $\mathrm{CO}_{2}$ displacement, vertical permeability may be important in oil recovery. The vertical movement of $\mathrm{CO}_{2}$ in reservoirs is controlled by the vertical permeability of the formation. In order to explore the impact of vertical permeability in oil recovery, vertical permeabilities of $5 \mathrm{md}, 10 \mathrm{md}$, and $20 \mathrm{md}$ were simulated. Based on this analysis, there was a clear tendency that decreasing vertical permeability increases oil recovery and reduces the $\mathrm{CO}_{2}$ utilization rate. In short, the miscible $\mathrm{CO}_{2}$ flooding process favors reservoirs with low vertical permeability because the overriding effect of carbon dioxide is diminished.

\section{Well Spacing}

To investigate the impact of well spacing (pattern size), the pattern area was reduced from the 160 acres of the base case to 80 acres and then to 40 acres. In order to keep the same volume ratio of $\mathrm{CO}_{2}$ injected per barrel of oil, the total $\mathrm{CO}_{2}$ injection for the 80 acre model was adjusted to $2 \mathrm{MMscf} / \mathrm{d}$, half of the 160 acre model. A total amount of $1 \mathrm{MMscf} / \mathrm{d}$ of $\mathrm{CO}_{2}$ was injected into the 40 acre model. From the analysis, miscible carbon dioxide flooding favors large well spacing patterns because they yield a better efficiency, require fewer wells, and still utilize similar volumes of $\mathrm{CO}_{2}$.

\section{Oil Viscosity}

In miscible $\mathrm{CO}_{2}$ injection, carbon dioxide is mixed with reservoir oil. This miscible process greatly reduces the oil viscosity. Carbon dioxide flooding has generally been applied to low oil viscosities. In order to investigate the effect of oil viscosity in carbon dioxide floods, the oil viscosity was increased to $4 \mathrm{cp}$ and $40 \mathrm{cp}$. The miscibility pressure was assumed constant at 4,800 psia. This is a major assumption that limits the applicability of the results; however, it does provide some insight on the ability to apply miscible carbon dioxide displacement to high viscosity oils.

In general, the difference in oil recovery was very small when varying the oil viscosity. The maximum deviation of recovery factor was 3\%. Based on this limited study, oil viscosity itself affects ultimate recovery only slightly. This suggests that low gravity oil reservoirs may be favorable for miscible carbon dioxide flooding. The effect of viscosity needs additional study before drawing definite conclusions but these results indicate the potential of miscible $\mathrm{CO}_{2}$ flooding to viscous oil reservoirs.

\section{Injection Rates}

To maintain miscible pressures, $\mathrm{CO}_{2}$ injection must balance reservoir production. Injection of additional $\mathrm{CO}_{2}$ might affect the recovery. It makes sense that increasing the $\mathrm{CO}_{2}$ injection rate results in faster oil recovery. To investigate how the ultimate recovery is affected by the injection rate, the simulated $\mathrm{CO}_{2}$ injection rate was increased to $6 \mathrm{MMscf} / \mathrm{d}$ and then to 8 MMscf/d and compared to the base case model injection rate of $4 \mathrm{MMscf} / \mathrm{d}$.

From this work, it appeared that miscible $\mathrm{CO}_{2}$ floods favor high $\mathrm{CO}_{2}$ injection rates. The increase in injection rate yielded a better recovery efficiency, recovered oil more quickly, and still utilized essentially the same amount of $\mathrm{CO}_{2}$ per barrel of recovered oil. 


\section{Formation Thickness}

Technically, thin pay zones would appear to favor $\mathrm{CO}_{2}$ floods since they diminish the tendency of gravity override. However, the thicker zones have the advantage in oil volume. A detailed investigation was undertaken to determine which factor, gravity segregation or oil volume, is more significant. The formation thickness of the reservoir was modified from $30 \mathrm{ft}$ of the base case to $20 \mathrm{ft}$ and $40 \mathrm{ft}$. For comparison purposes, the injection rate was also adjusted proportionally to the models' pore volume to keep the same ratio of $\mathrm{CO}_{2}$ injection per barrel of oil. From a recovery efficiency viewpoint, it is reasonable to say that the carbon dioxide injection performs better in reservoirs that have thin pay zones. In miscible $\mathrm{CO}_{2}$ floods, minimizing gravity override is an advantage for increasing the recovery factor.

This conclusion is not to imply that the thicker reservoirs are not suitable candidates for miscible carbon dioxide flooding as the economics of recovering larger oil volumes may be more important than larger recovery factors.

\section{Summary}

From the results of the cases studied this research, the following conclusions are drawn:

1. The modified inverted nine-spot pattern yields the highest sweep efficiency for miscible $\mathrm{CO}_{2}$ floods, compared to the five-spot, the nine-spot, and the inverted nine-spot. Up to an additional $10 \%$ of remaining oil in place can be recovered by the modified inverted ninespot relative to the five-spot pattern.

2. Increasing the $\mathrm{CO}_{2}$ injection rate improves the recovery efficiency. The recovery factor increases roughly $6 \%$ when doubling the carbon dioxide injection rate from $4 \mathrm{MMscf} / \mathrm{d}$ to $8 \mathrm{MMscf} / \mathrm{d}$.

3. Miscible carbon dioxide floods favor reservoirs that have low vertical permeability as it minimizes the effect of gravity segregation.

4. The completion profile of the wells can significantly affect recovery. It appears the best completion technique is to perforate wells only at the bottom intervals of the pay zone. Oil recovery is substantially increased approximately $15 \%$ of remaining oil in place by using the bottom perforation model compared to perforating the entire interval.

5. Miscible carbon dioxide flooding favors large well spacing patterns because they yield a better efficiency, require less investment in drilling wells, and still utilize the same amount of $\mathrm{CO}_{2}$.

6. Ignoring miscibility pressure, oil viscosity appears to have a minor impact on oil recovery in the miscible $\mathrm{CO}_{2}$ injection process. While needing additional study, this suggests heavy oil reservoirs may be viable candidates for miscible $\mathrm{CO}_{2}$ flooding.

7. Gravity override is significantly diminished in thin formations. As a result, the miscible $\mathrm{CO}_{2}$ displacement process seems to be more efficient in reservoirs that have relatively thin pay zones. The recovery factor is nearly 5\% higher in the thin reservoirs studied. However, one cannot overlook the economics of additional oil volume in place for the thicker reservoirs.

Based on the above analysis of the simulation runs, a methodology was proposed for operating miscible carbon dioxide floods. This methodology can provide help to enhance oil recovery in active carbon dioxide projects. In addition, it also can serve as a strategy for 
designing new carbon dioxide floods in the future. The following operating strategies may have application and should be considered.

1. Adjust the well pattern to the modified inverted-nine spot pattern.

2. Modify the perforation profile to the bottom intervals of the reservoir.

3. Maximize the $\mathrm{CO}_{2}$ injection rate without fracturing the formation.

4. Increase the well spacing of the operating patterns as much as possible.

\section{Subtask 2.2 Evaluation of Heavy Oil Displacement by $\mathrm{CO}_{2}$ Flooding}

There has been a considerable amount of literature dedicated to research on carbon dioxide flooding on light crudes and miscible processes. The application of $\mathrm{CO}_{2}$ immiscible flooding on heavy oils has received less attention although there have been several laboratory and field studies conducted. Laboratory studies generally concentrated on core flood experiments with different compositions of crude, variations in $\mathrm{CO}_{2}$ floods, and modifications to the slug size during a flood (Jha, 1985; Rojas and Ali, 1986). Most of the simulation work done on immiscible/miscible $\mathrm{CO}_{2}$ flooding has been done as part of field studies (Moffitt and Zornes, 1992; Reid and Robinson, 1981; Hatzignatiou and Lu, 1994; Spivak and Chima, 1984), but their numbers have been relatively few.

This project evaluates heterogeneity and viscous effects by performing a reservoir simulation study on the Wash-Fred formation in the Martinville field, Mississippi, in which a premature breakthrough of $\mathrm{CO}_{2}$ was observed. The purpose of this simulation study is to understand the mechanisms which lead to an early gas breakthrough and study various mitigating techniques from these simulations. Using the results from an approximate history match will allow us to evaluate and propose different methods which could help to overcome the problem effectively. Mitigating techniques we intend to study are WAG processes, surfactant injection (foam flood) and a gelled $\mathrm{CO}_{2}$ flood.

The Wash-Fred formation in the Martinville Field, Simpson County, Mississippi occurs at a depth of $8600 \mathrm{ft}$ (measured depth). This formation is divided into two zones, the Upper Wash-Fred and the Lower Wash-Fred. The current project studies only the Upper Wash-Fred formation (also referred to as Wash-Fred-8500 sand). Table 3 provides a list of reservoir properties for the formation.

The Wash-Fred formation first started production in October 1997 from the well MFU 15-16 \#1. Later MFU 15-9 \#1 was drilled to determine the oil-water contact. Finally, the MFU 14-13 \#1 was drilled. Initial mapping indicated that the MFU 14-13 \#1 would be at a structural high position, but after drilling the well the reservoir was remapped with the MFU 15-16 \#1 structurally high. Apart from these wells, MFU 22-1 \#2, MFU 14-13 \#1, MFU 22-7 \#1 also produce from this zone. After nearly six years of producing oil from the Wash-Fred reservoir on aquifer water drive, one of the up-dip wells (the MFU 15-16 \#1) was converted to a $\mathrm{CO}_{2}$ injector. Since the wells were not in any traditional spatial pattern, injection was designed with an idea of trapping oil between $\mathrm{CO}_{2}$ at the top and a strong water drive at the bottom, thereby enabling an easier production of oil from the down-dip wells. Only two months after $\mathrm{CO}_{2}$ injection began, breakthrough was observed in the well nearest the injector (the MFU 22-1 \#2). Due to this premature breakthrough, $\mathrm{CO}_{2}$ injection had to be curtailed and later stopped. Injection began from another well (MFU 15-9\#1) down-dip in the formation and is currently the only $\mathrm{CO}_{2}$ injector in the Wash Fred. 


\begin{tabular}{|c|c|}
\hline Depth & 8500 \\
\hline Oil Gravity & $14^{\circ} \mathrm{API}$ \\
\hline GOR & $50 \mathrm{scf} / \mathrm{STB}$ \\
\hline $\mathrm{B}_{\mathrm{o}}$ & $3900 \mathrm{psig}$ \\
\hline BHP & $198^{\circ} \mathrm{F}$ \\
\hline BHT & $26.0 \%$ \\
\hline Porosity & $39.0 \%$ \\
\hline Water Saturation & $3.05 \mathrm{RB} / \mathrm{STB}\left(@ \mathrm{bubble}=\mathrm{P}_{\mathrm{i}}\right)$ \\
\hline Permeability & 6125 \\
\hline Average Net pay & $7.3 \mathrm{MMBO}$ (from Side wall core study) \\
\hline Volume (acre-ft) & $27.4 \%$ \\
\hline OOIP & $35 \%$ \\
\hline Primary + Secondary recovery &
\end{tabular}

Table 3: Reservoir properties of Wash Fred 8500

This study focuses on the section of the field where rapid breakthrough occurred between an injector-producer pair. A detailed fluid characterization model was built to best represent the reservoir fluid in order to study the fluid properties and its effect on the early breakthrough. Next, a 2-D reservoir model was built to represent the flow between the injector-producer pair. The model was constructed based on the theoretical behavior of streamlines between an injector and producer in a homogeneous reservoir in order to try to capture volumetric aspects to the displacement.

Denbury Resources, Inc. provided laboratory measured data from a fluid composition analysis from the MFU 22-1 \#2 well. The compositional analysis of the gas had 18 components while the stock tank oil composition had 41 components. The detailed compositional analyses were then recombined to generate a phase diagram and minimum miscibility pressure value using WinProp $^{\circledR}$ software package from the Computer Modeling Group (CMG). The WinProp ${ }^{\circledR}$ options used were "Recombination of separator oil and gas", "Lumping of components" and "Phase diagram construction". The resulting compositional system had 40 of the original components and $\mathrm{a}_{36+}$ pseudo-component. An 8 psuedo-component fluid system was chosen as the right balance between simulation model speed and fluid behavior accuracy for the simulation model. An iterative process was initiated to select an appropriate lumped set of the 8 psuedocomponents that matched not only the detailed compositional results, but also other data provided by DRI such as oil swelling tests, viscosity data and slim tube miscibility simulations.

After each trial, the phase diagram was plotted and compared to the 40 component phase diagram and to the swelling data and viscosity data. This process was repeated until a satisfactory match was obtained. Table 4 shows the lumping scheme for which the best match was observed.

Figure 16 shows the P-T phase diagrams for the 40 component and the eight pseudocomponent systems while Figure 17 shows a comparison of the match to the swelling test data and to the viscosity data from DRI. Minimum Miscibility Pressure values were computed to be 7275 psi for the 40 component system, 7590 for the 8 pseudo-component system and 7240 psi for the simulated slim tube experiment using the 8 pseudo-component fluid system.

The Wash Fred reservoir is a small reservoir with an estimated Original Oil In Place (OOIP) of 7.34 MMSTB. This reservoir has a strong water drive mechanism which helped maintain pressure during the primary production phase which lasted from October, 1997 


\begin{tabular}{|c|c|}
\hline Pseudo Component & Mole Fraction (\%) \\
\hline $\mathrm{N}_{2}$ & 0.4577 \\
\hline $\mathrm{CO}_{2}$ & 0.1414 \\
\hline $\mathrm{C}_{1}$ & 14.1641 \\
\hline $\mathrm{C}_{2}-\mathrm{C}_{3}$ & 1.2220 \\
\hline $\mathrm{C}_{4}-\mathrm{C}_{5}$ & 1.3408 \\
\hline $\mathrm{C}_{6}-\mathrm{C}_{12}$ & 16.1274 \\
\hline $\mathrm{C}_{13}-\mathrm{C}_{35}$ & 51.3537 \\
\hline $\mathrm{C}_{36}{ }^{+}$ & 15.3202 \\
\hline
\end{tabular}

Table 4: Lumping and mole fractions of the 8 psuedo-component system

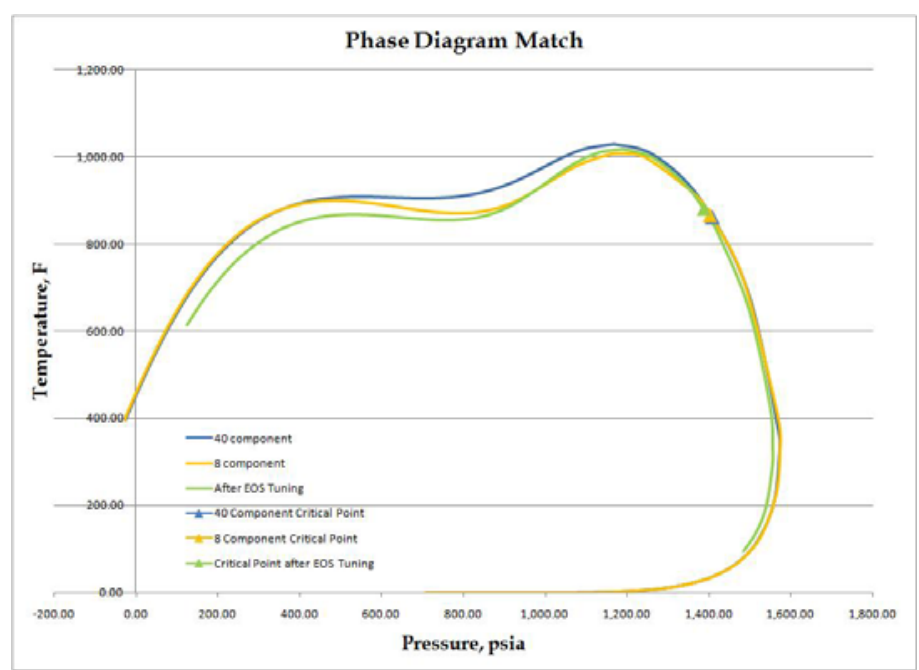

Figure 16: Phase diagram of the Wash-Fred crude for the 40 component and 8 component lumped system.
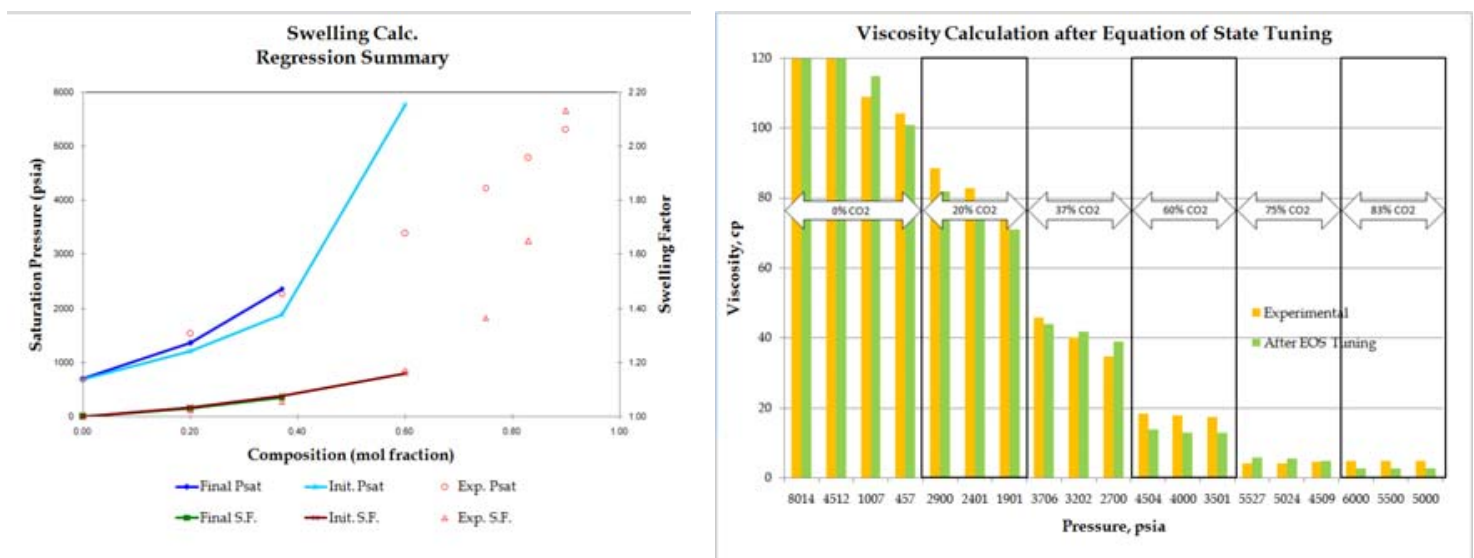

Figure 17: Swelling test and Viscosity comparison. 
to March, 2006. During this period approximately 1.61 MMSTB of oil was produced along with 12.7MMSTB of water. Figure 18 shows the structure map of the Wash Fred - 8500 formation.

To get an estimate of average reservoir pressure at the time $\mathrm{CO}_{2}$ injection had begun, a material balance calculation was done and an average reservoir pressure of 3650 psia was predicted. This suggests a very small drop in reservoir pressure of 250 psia, over a period of 8 years. Material Balance also pointed towards a large quantity of water encroachment into the reservoir of approximately 13.9 million barrels.

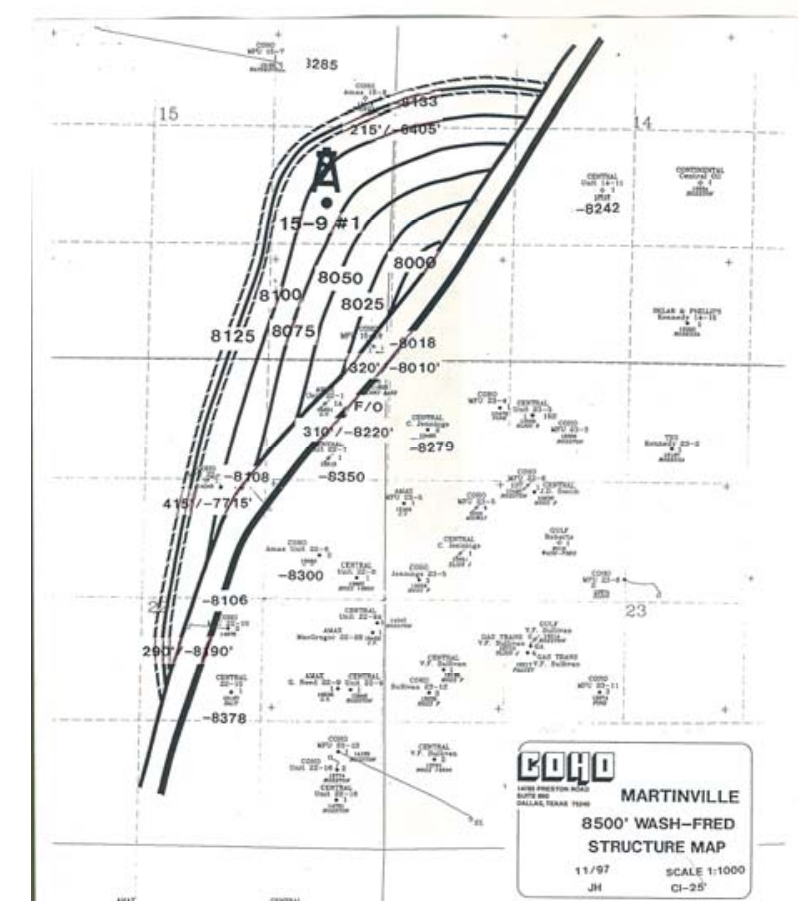

\section{Figure 18: Structure map of the Wash Fred 8500 formation, Martinville field (from Denbury Resources, Inc.)}

Prior to implementation, Denbury Resources, Inc. performed a full-scale field simulation for this field where various options of injecting $\mathrm{CO}_{2}$ from different wells such as updip $\mathrm{CO}_{2}$ injection or downdip $\mathrm{CO}_{2}$ injection were studied along with their impact on eventual oil recoveries and sweep. The objective of the current study, however, was not to build a full field scale reservoir model but to build a representative model of the reservoir between the injector (MFU 15-16\#1) and producer where early gas breakthrough was observed (MFU 22-1\#2). This should enable us to somewhat study the causes of this early gas breakthrough but also evaluate possible mitigation techniques.

Figure 19 and Figure 20 show the reservoir model built for this study with a first estimate of layer permeability values using data from a single core from the field. The dip angel is similar to that in the reservoir and the tapered area tries to approximate the streamlines for an ideal system to obtain appropriate velocities away from the wells in the $2 \mathrm{D}$ model. The model has $100 \times 1 \times 30$ grid blocks with grid dimensions of $11^{\prime}$ in the $\mathrm{x}$-direction, $3{ }^{\prime}$ in the $\mathrm{z}$-direction and a variable grid dimension in the y-direction. The permeability distribution shown in this model is arrived at by calculating a simple average of the side-wall core permeabilities provided by 


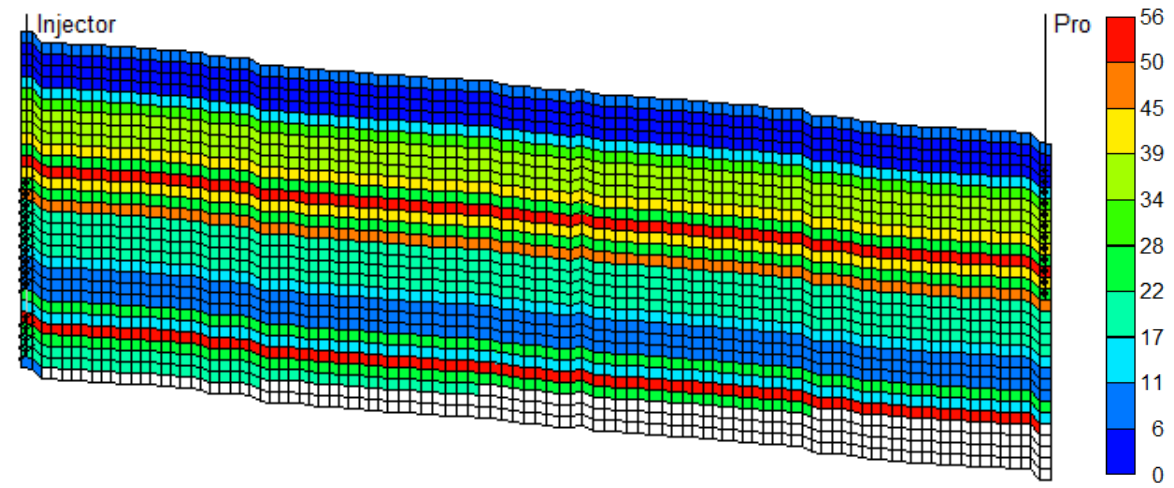

Figure 19: $\mathrm{X}-\mathrm{Z}$ cross-sectional view of the reservoir model (colors are permeability, $\mathrm{mD}$ )

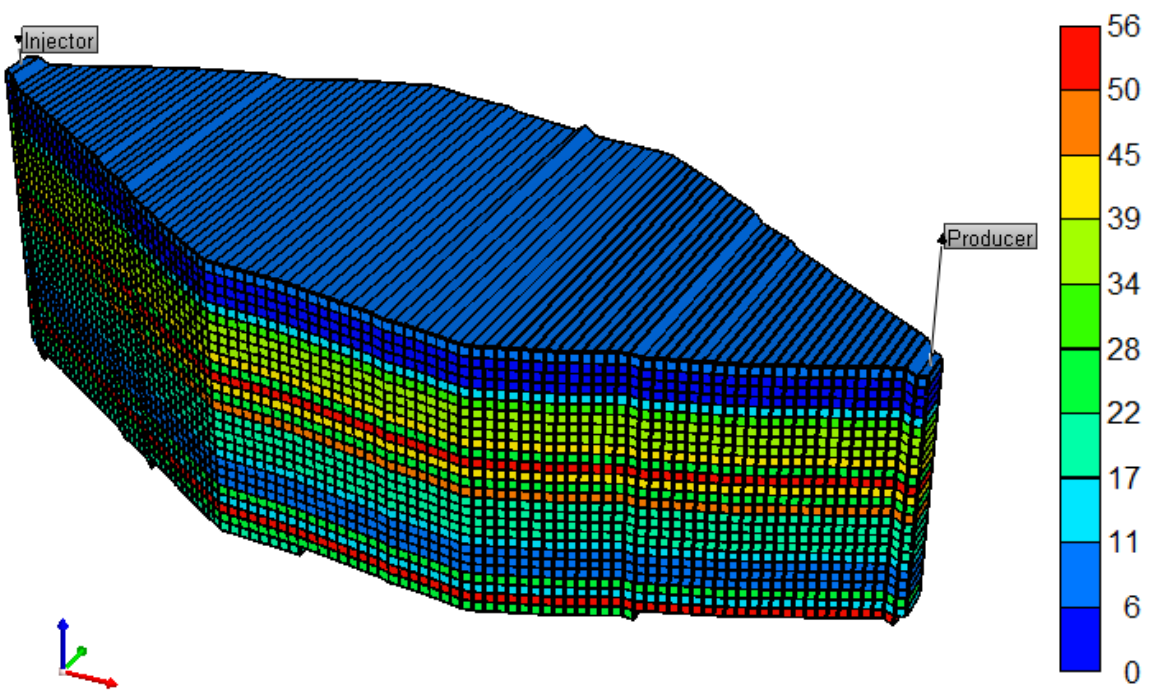

Figure 20: 3-D view of the reservoir model (colors are permeability, mD)

Denbury Resources, Inc. These permeability values are assumed to be constant over the whole distance between the injector and producer. Similar calculations of simple average of side-wall core data were used to fill the porosity values in all the grid blocks. For initial grid permeability values, $\mathrm{k}_{\mathrm{v}}=\mathrm{k}_{\mathrm{h}} / 10$ was used to populate permeabilities in the vertical direction. All parameters will be uncertainties to manipulate in both the history match as well as the sweep improvement strategies.

The task now is to simulate the problem of early $\mathrm{CO}_{2}$ breakthrough, which is the focal point of this study. Once an approximate match to the historical data is attained, mitigating techniques to prevent early $\mathrm{CO}_{2}$ breakthrough will be investigated. Mitigating techniques to be studied are the water alternating gas (WAG) strategy, surfactant (foam) flooding and some form of viscosity increase technique - typically a polymer gel to increase $\mathrm{CO}_{2}$ viscosity. Although it might be too late to apply these EOR techniques to the Wash-Fred reservoir, it should provide insight into how the Wash-Fred would have behaved to these techniques. Moreover, it will serve as a documentation of these EOR methods which could be applied on similar reservoirs elsewhere. 


\section{Conclusions and Recommendations}

In this work we have investigated a number of very disparate methods primarily to evaluate sweep efficiency for $\mathrm{CO}_{2}$ flood operations. These techniques have ranged from inexpensive mainly data evaluation techniques to relatively expensive cased hole saturation logging and reservoir simulation studies. Our overriding view of the evaluation and mitigation techniques discussed has been that an integrative approach using the available data or data that is reasonably easy to acquire will provide valuable insight in the design of the next steps in improving recovery from a field. The most important design parameter in any flood is the cost of the displacing or enhancement fluid. Understanding how much of the fluid is needed only comes from an understanding of where the remaining oil resides in the reservoir and whether the enhancement fluid can contact that oil or allow additional enhancement fluid to contact the oil. Using that philosophy and based on the work presented in this report, our conclusions are:

- The "post-mortem" analysis of Little Creek showed that an understanding of vertical and areal heterogeneity is crucial for understanding sweep processes as well as understanding appropriate mitigation techniques to improve the sweep

- The combination of relatively inexpensive production and/or injection logs with the more expensive cased-hole saturation measurement tools proposed in this work is an excellent way to determine the near well distribution of fluids. These tools can provide some understanding of vertical heterogeneity when core data is in short supply or not available

- Completion techniques should be modified to take advantage of the primary mechanisms controlling flow behavior. Completing injectors and producers low in the formation was found to be successful for homogeneous systems dominated by gravity. It was also found that recovery can be improved by ensuring that a well is fully completed so $\mathrm{CO}_{2}$ can contact the entire reservoir for those cases where heterogeneity dominates.

- Horizontal completions and using the GAGD process also can be beneficial even in cases with low structural relief. However, care must be taken for these cases since gravity forces are less significant and slight changes in well placement may have a large impact on recovery.

- Combining the near well distribution of fluids with simple statistical or numerical approaches for evaluating the connections between injectors and producers provides a relatively inexpensive way to evaluate the areal distribution of fluids in the reservoir. Evaluating the connectivity between injector and producer well pairs is slightly more complicated for $\mathrm{CO}_{2}$ floods than waterfloods due to the higher compressibility of the $\mathrm{CO}_{2}$. This likely means that successful use of these tools in a $\mathrm{CO}_{2}$ flood may require higher frequency data than may be available. In addition there is higher uncertainty in the measurement of the produced fluids especially when recycling efforts are in progress.

- Using all of this information to drive a thorough, well-documented reservoir simulation study integrates the full sequence of issues dealt with in this work and should provide sufficient details on both the areal and vertical distribution of fluids

- Based on admittedly preliminary work, it does appear that experimental work using both analytic (laboratory) and field cores and fluids can be tied to field-scale processes, but care must be taken to evaluate the mechanism the enhancement fluid takes to improve recovery and the distribution of fluids in the reservoir 


\section{References}

Albertoni, A., Infering interwell connectivity from well-rate fluctuations in waterfloods, M.S. Thesis, The University of Texas at Austin, May 2002.

Amadi, S., and Hughes, R.G.: "Evaluation of Behind-Pipe Saturation in a Miscible $\mathrm{CO}_{2}$ Flood", SPE 113887 in Proceedings of the 2008 SPE Improved Oil Recovery Symposium, Tulsa, Oklahoma, U.S.A., 19-23 April 2008.

Bassiouni Zaki: Theory, Measurement, and Interpretation of Well Logs, SPE Textbook Series Volume 4, 1994

Bortkevitch, S.V., Kostrov, S.A., Savitsky, N.V. and Wooden, W.O.: "Method and Apparatus For Enhanced Oil Recovery by Micro-Disperesed Gas-Liquid Mixture into Oil Bearing Formation", US Patent No. 20050077636, April 14, 2005.

Caudle, B.H. and Dyes, A.B., "Improving Miscible Gas Dispalcemnt by Gas-Water Injection" Trans. AIME, 1958. 213: p. 281-284.

Chatfield, C.: Statistics for Technology: A Course in Applied Statistics, Chapman \& Hall, 1983.

Computer Modelling Group: IMEX Users Manual, 2007

*Cottrell, C.W.: "The Sensitivity of the History Match of the Little Creek $\mathrm{CO}_{2}$ Pilot to Reservoir Characteristics/Simulation Parameters and Their Effect on Pattern Flood Performance," Shell Western E\&P Inc. Internal Report LC84059002W, June, 1984.

Cronquist, C.: "Waterflooding by Linear Displacement in Little Creek Field, Mississippi," Journal of Petroleum Technology, May, 1968.

Dinh, A., Reservoir Characterization for the Delaware-Childers Field Based on Flow Rate Analysis, M.S. Thesis, The University of Oklahoma at Norman, 2003.

Dinh, A. and Tiab.D, "Inferring interwell connectivity from well bottomhole pressure fluctuations in waterfloods", SPE Journal, October, 2008.

Dykstra, H. and Parsons, R.L.: "The Prediction of Oil Recovery by Water Flood", In: A.P. Institute (Editor), Secondary Recovery of Oil in the United States, New York, pp. 160-174, 1950.

Edwards A.L, An Introduction to Linear Regression and Correlation, $2^{\text {nd }}$ Edition. NewYork: W.H. Freaman, 1984

*Fair, W.B.: "The Effect of Little Creek Field Reservoir Properties on $\mathrm{CO}_{2}$ Miscible Flooding," Shell Offshore Inc. Coastal Division, July, 1987. 
Green, D.W. and Willhite, G.P.: Enhanced Oil Recovery, SPE Textbook Series, No. 6, Society of Petroleum Engineers, 1998.

Hansen, P.W.: “A CO2 Tertiary Recovery Pilot Little Creek Field, Mississippi," SPE paper 6747 presented at the 52nd Annual Technical Conference and Exhibition, Denver, CO, 1977b.

*Hansen, P.W.: "A CO 2 Tertiary Recovery Pilot Study and Field Expansion $\mathrm{CO}_{2}$ Process Analysis," Shell Oil Company Internal Report, Onshore Division, June, 1977a.

Hatzignatiou, D. G. and Lu, Y.: "Feasibility study of CO2 Immiscible Displacement Process in Heavy Oil Reservoirs", 45th Annual Technical Meeting of Petroleum Society of CIM, Calgary.

Jha, K.N.: "A Laboratory Study of Heavy Oil Recovery with Carbon Dioxide", Journal of Canadian Petroleum Technology, March-April, 1986, pp. 54-63.

Jensen, J.L., Lake, L.W, Corbett, P.W.M. and Goggin, D.J., Statistics for Petroleum Engineers and Geoscientists, Elsevier, 2000.

Kulkarni, M.M., Immisible and Miscible Gas-Oil Displacement in Porous Media, MS Thesis, 2003, Louisiana State University: Baton Rouge.

Kuuskraa, V.A., "Maximizing Oil Recovery Efficiency and Sequestration of $\mathrm{CO}_{2}$ with Game Changer $\mathrm{CO}_{2}$-EOR Technology," SPE Distinguished Lecturer Series, January, 2008.

Lee, W.J.: Well Testing, Society of Petroleum Engineers of AIME, 1982.

Lovy, D., WinDIG Version 2.5, 1996

Mahmoud, T.N. and Rao, D.N.: "Mechanisms and Performance Demonstration of the GasAssisted Gravity-Drainage Process Using Visual Models," SPE paper 110132 presented at the 2007 SPE Annual Technical Conference and Exhibition, Anaheim, California, 11-14 November, 2007.

Moffitt, P. D. and Zornes, D. R.: "Postmortem Analysis: Lick Creek Meakin Sand Unit Immiscible CO2 Waterflood Project", SPE paper 24933 presented at the 67th Annual Technical Conference and Exhibition, Washington DC, 4-7 October, 1992.

*Morse, E.W.: "Simulation Studies Toward Matching the Little Creek $\mathrm{CO}_{2}$ Pilot and Predicting Phase 1 Performance," Shell Oil Company Internal Technical Progress Report BRC 73-82, November, 1979.

Müller, N., Ramakrishnan, T.S., Boyd, A. And Sakruai, S.: "Time-lapse carbon dioxide monitoring with pulsed neutron logging", International Journal of Greenhouse Gas Control I, June, 2007. 
North, R. J.: "Through-Casing Reservoir Evaluation Using Gamma Ray Spectroscopy", Paper SPE 16356, presented at the SPE California Regional Meeting, Ventura, California, April, 1987

Ogunyomi, G.: Evaluation Of The Interwell Connectivity Of Little Creek Field, Mississipi From Production Data, MS Thesis, 2009, Louisiana State University: Baton Rouge.

*Orr, F.M.: "Phase Behavior and Properties of mixtures of $\mathrm{CO}_{2}$ and Little Creek Crude Oil," Shell Development Company Bellaire Research Center, BRC, Houston, November, 1976.

Pennell, S.P., Denbury Resources, Inc., Personal Communication, July, 2007.

Rao, D.N., Ayirala, S.C., Kulkarni, M.M. and Sharma, A.P.: "Development of Gas Assisted Gravity Drainage (GAGD) Process," SPE paper 89357 presented at the Fourteenth SPE/DOE Improved Oil Recovery Symposium, Tulsa, OK, 17-21 April, 2004.

Rapoport, L.A. and W.J. Leas, "Properties of Linear Waterfloods". Trans. AIME 1953, Society of Petroleum Engineers.

Reid, T. B. and Robinson, H. J: "Lick Creek Meakin Sand Unit Immiscible CO2/Waterflood Project”, Journal of Petroleum Technology , September, 1981, pp. 1723-1729.

Rojas, G. and Farouq Ali, S. M.: "Scaled model studies of carbon dioxide/brine injection strategies for heavy oil recovery from thin formations", Journal of Canadian Petroleum Technology , January-February, 1986, pp. 85-94.

Sayarpour,M., Kabir, C.S, Lake, L. W., "Field Applications of capacitance-resistive models in waterfloods", Society of Petroleum Engineers, paper SPE 114983, 2008.

Schlumberger: Log Interpretation; Volume 1 - Principles, 1972

Senocak, D.: Evaluation Of Sweep Efficiency Of A Mature Co2 Flood In Little Creek Field, Mississippi, MS Thesis, 2008, Louisiana State University: Baton Rouge.

Senocak, D., Hughes, R.G., Pennell, S.P. and Gibson, C.E.: "Effective Use of Heterogeneity Measures in the Evaluation of a Mature $\mathrm{CO}_{2}$ Flood," SPE paper 113977 presented at the Sixteenth SPE/DOE Improved Oil Recovery Symposium, Tulsa, OK, 19-23 April, 2008.

Serra, O.: Well Logging -Data Acquisition and Applications, Elsevier, 2004

*Shannon, M.T.: "Petrophysical Review - Little Creek Field Lincoln and Pike Counties, Mississippi," Shell Western E\&P Inc. , Onshore East Division, January, 1984.

*Smith, D.A.: "Lower Tuscaloosa Geology and Petrophysics Relating to a Carbon Dioxide Miscible Project," Shell Oil Company Internal Report, New Orleans, LA, June, 1973. 
Smolen J.: Cased Hole and Production Log Evaluation, Pennwell Publishing Company, Tulsa, Oklahoma, 1996

Spivak, A. and Chima, C. M.: "Mechanisms of Immiscible CO2 Injection in Heavy Oil Reservoirs, Wilmington Field, CA" paper SPE 12667 presented at the 1984 California Regional Meeting, Long Beach, CA, 11-13 April, 1984.

Svor, T. R., and Globe, M. P.: "A Three-Phase Quantitative Monitoring for $\mathrm{CO}_{2}$ Flood" Paper SPE/DOE 10684 presented at the Third SPE/DOE Joint Symposium on Enhanced Oil Recovery, Tulsa, OK, April, 1982.

Todd, M.R. and Longstaff, W.J.: "The Development Testing and Application of a Numerical Simulator for Predicting Miscible Flood Performance," Journal of Petroleum Technology: pp. 874-882, July, 1972.

Tran, N.T.: Evaluation And Enhancement Of Miscible Carbon Dioxide Flooding, MS Thesis, University of Oklahoma, Norman, 2005.

Watters, R.L., Jr., Chemistry WebBook of National Institute of Standards and Technology, http://webbook.nist.gov/chemistry/fluid/.

Werren, E.G., Shew, R.D., Adams, E.R. and Stancliffe, R.J.: "Meander-Belt Reservoir Geology, Mid-Dip Tuscaloosa, Little Creek Field, Mississippi", Sandstone Petroleum Reservoirs: New York, Springer- Verlag, pp. 85-105, 1990.

Youmans, A. H., Hopkinson, E. C., Bergan, R. A., and Oshry, H. H. : "Neutron Lifetime. A New Nuclear Log", Journal of Petroleum Technology, March, 1964, pg 319-328

Youngren, G.K. and Charlson, G.S.: "History Match Analysis of the Little Creek $\mathrm{CO}_{2}$ Pilot Test," Journal of Petroleum Technology: pp. 2042-2052, November, 1980.

Youseff, A.A., Investigating Statistical Techniques to Infer Interwell Connectivity From Production and Injection Fluctuations, PhD Dissertation, The University of Texas at Austin, 2006.

\footnotetext{
* Internal Shell Oil Company Report obtained from Denbury Resources, Inc. well files
} 